\title{
A BIJECTION BETWEEN NONCROSSING AND NONNESTING PARTITIONS FOR CLASSICAL REFLECTION GROUPS
}

\author{
ALEX FINK AND BENJAMIN IRIARTE GIRALDO
}

\begin{abstract}
We present an elementary type preserving bijection between noncrossing and nonnesting partitions for all classical reflection groups, answering a question of Athanasiadis.
\end{abstract}

\section{INTRODUCTION AND BACKGROUND}

The Coxeter-Catalan combinatorics is an active field of study in the theory of Coxeter groups. Several diverse and independently motivated sets of objects associated to a Coxeter group $W$ have the cardinality $\prod_{i=1}^{r}\left(h+d_{i}\right) / d_{i}$, where $h$ is the Coxeter number of $W$ and $d_{1}, \ldots, d_{r}$ its degrees. At the core of the Coxeter-Catalan combinatorics are the problems of explaining these equalities of cardinalities. Two of the sets of objects involved are

- the noncrossing partitions $N C(W)$, which in their classical (type $A$ ) avatar are a long-studied combinatorial object harking back at least to Kreweras 12, and in their generalisation to arbitrary Coxeter groups are due to Bessis and Brady and Watt [5], 7]; and

- the nonnesting partitions $N N(W)$, introduced by Postnikov 14 for all the classical reflection groups simultaneously.

Athanasiadis in [3] proved in a case-by-case fashion that $|N N(W)|=|N C(W)|$ for the classical reflection groups $W$, and asked for a bijective proof. This was later improved by Athanasiadis and Reiner 4 to a proof for all Weyl groups, cited as Theorem 1.20 below. This proof showed that nonnesting and noncrossing partitions are equidistributed by type, a statistic for partitions defined in ; but it handled the classical reflection groups in a nonuniform case-by-case fashion, and was not bijective for the exceptional groups.

Our contribution has been to provide a bijection which, given particular fixed choices of coordinates in the representation, works uniformly for the classical reflection groups. Our proof also provides equidistribution by type. The cases of our bijection for types $B, C$, and $D$ have not appeared before in the literature. The ultimate goal in connecting $N N(W)$ and $N C(W)$, a case-free bijective proof for all Weyl groups, remains open. The special nature of our choices of coordinates enables the construction of bump diagrams, and the present lack of a notion of bump diagrams for the exceptional groups would seem to be a significant obstacle to extending our approach.

The authors would like to thank Federico Ardila and the SFSU-Colombia initiative for their support in this research. 
Two other papers presenting combinatorial bijections between noncrossing and nonnesting partitions independent of this one, one by Stump [18] and by Mamede [13, appeared essentially simultaneously to it. Both of these limit themselves to types $A$ and $B$, and our approach is also distinct to them in its type preservation and in providing additional statistics characterising the new bijections. More recently Conflitti and Mamede 9] have presented a bijection in type $D$ which preserves different statistics to ours (namely openers, closers, and transients).

In the remainder of this section we lay out the definitions of the objects involved: in $\$ 1.1$, the uniform definitions of nonnesting and noncrossing partitions; in $\$ 1.2$, a mode of extracting actual partitions from these definitions which our bijections rely upon; in $\$ 1.3$ the resulting notions for classical reflection groups. In section 2 we present a type-preserving bijection between noncrossing and nonnesting partitions that works for all the classical reflection groups. We prove our bijection in a case by case fashion for each classical type, unpacking and specializing the definition to a more concrete bijection in each type in turn.

1.1. Uniform noncrossing and nonnesting partitions. For noncrossing partitions we follow Armstrong [1, $\$ 2.4-6]$. The treatment of nonnesting partitions is due to Postnikov [14].

Let $(W, S)$ be a finite Coxeter system of rank $r$, so that $S=\left\{s_{1}, s_{2}, \ldots, s_{r}\right\}$ generates the group

$$
W=\left\langle s_{1}, \ldots, s_{r}: s_{i}^{2}=\left(s_{i} s_{j}\right)^{m_{i j}}=1\right\rangle .
$$

We will always take the $m_{i j}$ finite. Let $T=\left\{s^{w}: s \in S, w \in W\right\}$ be the set of all reflections of $W$, where $s^{w}=w^{-1} s w$ denotes conjugation. Let $[r]=\{1, \ldots, r\}$. Consider the $\mathbb{R}$-vector space $V=\operatorname{span}_{\mathbb{R}}\left\{\alpha_{i}: i \in[r]\right\}$ endowed with the inner product $\langle\cdot, \cdot\rangle$ for which $\left\langle\alpha_{i}, \alpha_{j}\right\rangle=-\cos \left(\pi / m_{i j}\right)$, and let $\rho: W \rightarrow \operatorname{Aut}(V)$ be the geometric representation of $W$. This is a faithful representation of $W$, by which it acts isometrically on $V$ with respect to $\langle\cdot, \cdot\rangle$.

The set $N C(W)$ of (uniform) noncrossing partitions of $W$ is defined as an interval of the absolute order.

Definition 1.1. The absolute order $\mathrm{A} b s(W)$ of $W$ is the partial order on $W$ such that for $w, x \in W, w \leq x$ if and only if

$$
l_{T}(x)=l_{T}(w)+l_{T}\left(w^{-1} x\right),
$$

where $l_{T}(w)$ is the minimum length of any expression for $w$ as a product of elements of $T$. A word for $w$ in $T$ of length $l_{T}(w)$ will be called a reduced $T$-word for $w$.

The absolute order is a poset graded by $l_{T}$, with unique minimal element $1 \in W$. It has several distinguished maximal elements:

Definition 1.2. A standard Coxeter element of $(W, S)$ is any element of the form $c=s_{\sigma(1)} s_{\sigma(2)} \ldots s_{\sigma(r)}$, where $\sigma$ is a permutation of the set $[r]$. A Coxeter element is any conjugate of a standard Coxeter element in $W$.

All Coxeter elements have maximal rank in $\mathrm{A} b s(W)$.

Definition 1.3. Relative to any Coxeter element $c$, the poset of (uniform) noncrossing partitions is the interval $N C(W, c)=[1, c]$ in the absolute order. 
Although this definition appears to depend on the choice of Coxeter element $c$, the intervals $[1, c]$ are isomorphic as posets for all $c$ [1, Definition 2.6.7]. So we are free to use the notation $N C(W)$ for the poset of noncrossing partitions of $W$ with respect to any $c$.

Now assume $W$ is a Weyl group. The set $N N(W)$ of nonnesting partitions is defined in terms of the root poset.

Definition 1.4. The root poset of $W$ is its set of positive roots $\Phi^{+}$with the partial order $\leq$ under which, for $\beta, \gamma \in \Phi^{+}, \beta \leq \gamma$ if and only if $\gamma-\beta$ lies in the positive real span of the simple roots.

This definition of the root poset is distinct from, and more suited for connections to nonnesting partitions than, the one given in Björner \& Brenti [6], which does not require the Weil group condition, and which in fact is a strictly weaker order than Definition 1.4

Definition 1.5. A (uniform) nonnesting partition for $W$ is an antichain in the root poset of $W$. We denote the set of nonnesting partitions of $W$ by $N N(W)$.

To each root $\alpha$ we have an orthogonal hyperplane $\alpha^{\perp}$ with respect to $\langle\cdot, \cdot\rangle$, and these define a hyperplane arrangement and a poset of intersections.

Definition 1.6. The partition lattice $\Pi(W)$ of $W$ is the intersection poset of reflecting hyperplanes

$$
\left\{\bigcap_{\alpha \in S} \alpha^{\perp}: S \subseteq \Phi^{+}\right\} .
$$

Note that $\Pi(W)$ includes the empty intersection $V$, when $S=\emptyset$.

Now let $W$ be a classical reflection group, i.e. one of the groups $A_{r}, B_{r}, C_{r}$ or $D_{r}$ in the Cartan-Killing classification.

Each classical reflection group has a standard choice of coordinates which we will use throughout, that is an isometric inclusion of $V$ into a Euclidean space $\mathbb{R}^{n}$ bearing its usual inner product, not necessarily an isomorphism. This yields a faithful isometric representation $\rho^{\mathrm{cl}}: W \rightarrow \operatorname{Aut}\left(\mathbb{R}^{n}\right)$ of $W$, the superscript cl standing for "classical". In Section 2.10 of [10, a standard choice of simple roots is presented in the standard coordinates; our simple roots, in (1.1), are identical except that we've reversed the indexing, swapping $e_{1}, e_{2}, \ldots, e_{r}$ for $e_{r}, e_{r-1}, \ldots, e_{1}$.

$$
\begin{aligned}
& \Delta_{A_{r}}=\left\{e_{2}-e_{1}, e_{3}-e_{2}, \ldots, e_{r+1}-e_{r}\right\} \\
& \Delta_{B_{r}}=\left\{e_{1}, e_{2}-e_{1}, e_{3}-e_{2}, \ldots, e_{r}-e_{r-1}\right\} \\
& \Delta_{C_{r}}=\left\{2 e_{1}, e_{2}-e_{1}, e_{3}-e_{2}, \ldots, e_{r}-e_{r-1}\right\} \\
& \Delta_{D_{r}}=\left\{e_{1}+e_{2}, e_{2}-e_{1}, e_{3}-e_{2}, \ldots, e_{r}-e_{r-1}\right\}
\end{aligned}
$$

We will reserve $n$ for the dimensions of the particular coordinatisations presented here, writing $r$ when we mean the rank of $W$. Hence $n=r+1$ when $W=A_{r}$, but $n=r$ when $W$ is $B_{r}$ or $C_{r}$ or $D_{r}$. We will use the names $A_{n-1}, B_{n}, C_{n}, D_{n}$ henceforth.

Figure 1 exhibits the root posets of the classical reflection groups. We annotate the lower verges of the root posets with a line of integers, which for reasons of space we bend around the left side. Given a dot in Figure 1, if $i$ and $j$ are the integers in line with it on downward rays of slope 1 and -1 respectively, then it represents the root $\alpha=e_{j}-e_{i}$, where $e_{-k}=-e_{k}$ for $k<0$ and $e_{0}=0$. 

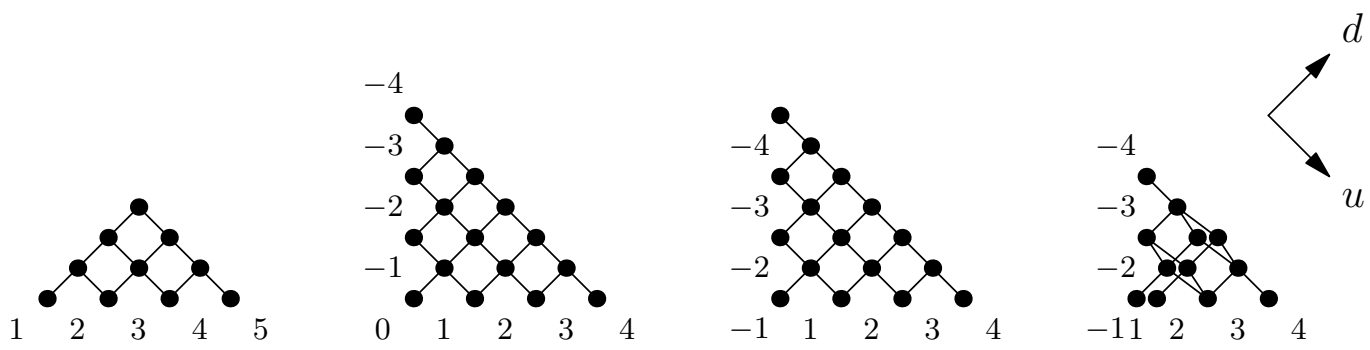

Figure 1. The root posets for groups (left to right) $A_{4}, B_{4}, C_{4}$, and $D_{4}$.

1.2. Classical partitions. The definitions of partitions matching the objects considered in classical combinatorics are framed geometrically in a way that has not been generalised to all Weyl groups, depending crucially as they do on the form the reflections take in the standard choice of coordinates. Our treatment of partitions and our drawings are taken from Athanasiadis and Reiner [4]. We have reversed the orderings of the ground sets from Athanasiadis and Reiner's presentation.

Let $W$ be a classical reflection group. The procedures to obtain objects representing $N N(W)$ and $N C(W)$ can be unified to a significant degree - though there will still be cases with exceptional properties - so we will speak of classical partitions for $W$.

Definition 1.7. A partition $\pi$ of the set

$$
\Lambda=\left\{ \pm e_{i}: i=1, \ldots, n\right\} \cup\{0\},
$$

is a classical partition for $W$ if there exists $L \in \Pi(W)$ such that each part of $\pi$ is the intersection of $\Lambda$ with a fiber of the projection to $\pi$. We write $\pi=\operatorname{Part}(L)$.

We will streamline the notation of classical partitions by writing $\pm i$ for $\pm e_{i}$. Thus, a classical partition for $W$ is a partition of $\pm[n]=\{1, \ldots, n,-1, \ldots,-n, 0\}$ for some $n$, symmetric under negation. A classical partition always contains exactly one part fixed by negation, which contains the element 0 , namely the fiber over $0 \in L$. Since the position of 0 is predictable given the other elements, in many circumstances we will omit it altogether. If the block containing 0 contains other elements as well, we shall call it a zero block. Negating all elements of a block of a classical partition yields a block. The zero block is the only fixed point of negation, so the other blocks come in pairs of opposite sign.

For example, a typical classical partition might look like

$$
\{\{1,2\},\{-1,-2\},\{3,-7,-8\},\{-3,7,8\},\{5\},\{-5\},\{4,6,-4,-6,0\}\}
$$

in which $\{4,6,-4,-6,0\}$ is the zero block. This is the partition depicted in Figure 2

Given a minimal set of equations for $L$, each of which must be of the form

$$
s_{1} x_{i_{1}}=\cdots=s_{k} x_{i_{k}}(=0)
$$

where the $s_{i} \in\{+1,-1\}$ are signs, the classical partition can be read off, one block from each equation. To the above corresponds $\left\{s_{1} i_{1}, \ldots, s_{k} i_{k}\right\}$ if the $=0$ is not included, and $\left\{ \pm i_{1}, \ldots, \pm i_{k}, 0\right\}$ if it is.

In case $W=A_{n-1}, \rho^{\text {cl }}$ fixes the set of positive coordinate vectors $\left\{e_{i}\right\}$. So a classical partition for $W$ will be the union of a partition of $[n]$ and its negative, 


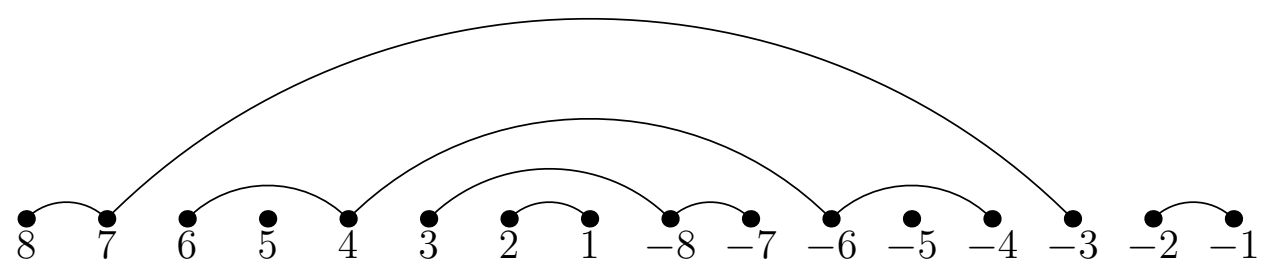

Figure 2. Example of a bump diagram of a noncrossing partition for $B_{8}=C_{8}$.

a partition of $-[n]$, with 0 in a block of its own. Here, and in everything we do henceforth with type $A$, we will omit the redundant nonpositive parts and treat type $A$ partitions as partitions of $[n]$.

In general the set $\left\{ \pm e_{i}\right\}$ is stabilized by $\rho^{\mathrm{cl}}$, giving rise to a faithful permutation representation of $W$. Combined with the notational efficacies of the last paragraphs, this is a convenient way to notate elements of $W$.

To exemplify this notation: for each classical reflection group we have a standard choice of Coxeter element $c$, obtained by taking the product of transpositions in the order they occur along the bottom of the standard diagram of the root system. Using the permutation representations they are

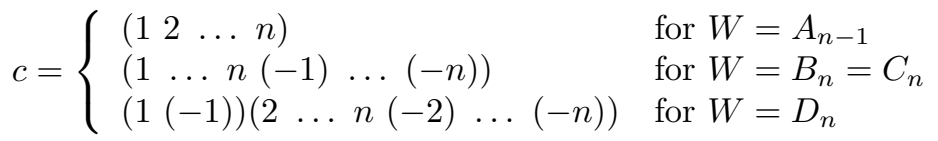

Finally, we introduce the type of a partition.

Definition 1.8. Let $\pi=\operatorname{Part}(L)$ be a classical partition for a classical reflection group $W$. The type type $(\pi)$ of $\pi$ is the conjugacy class of $L$ under the action of $W$ on $\Pi(W)$.

The collision of terminology between this sense of "type" and the sense referring to a family in the Cartan-Killing classification is unfortunate but standard, so we muddle along with it.

Combinatorially, the information captured in the type of a classical partition is related to the multiset of its block sizes. Given a classical partition $\pi$, let $\lambda$ be the cardinality of its zero block and $\mu_{1}, \ldots, \mu_{s}$ the cardinalities of the pairs of nonzero blocks of opposite sign. Then the partitions of the same type as $\pi$ are exactly those with zero block of size $\lambda$ and pairs of other blocks of sizes $\mu_{1}, \ldots, \mu_{s}$. The integer partition $\lambda$ which Athanasiadis in [3] calls the type of $\pi$ is the partition $\mu_{1}, \ldots, \mu_{s}$.

For example, a partition has the same type as the partition 1.2 ,

$$
\{\{1,2\},\{-1,-2\},\{3,-7,-8\},\{-3,7,8\},\{5\},\{-5\},\{4,6,-4,-6,0\}\},
$$

if its zero block of size 4 and it has three pairs of nonzero blocks with sizes 3,2 , and 1 .

1.3. Classical noncrossing and nonnesting partitions. Definitions of the classes of noncrossing and nonnesting classical partitions are perhaps most intuitively presented in terms of a diagrammatic representation, motivating the names "noncrossing" and "nonnesting". After Armstrong [1, §5.1] we call these bump diagrams.

Let $P$ be a partition of a totally ordered ground set $(\Lambda,<)$. 
Definition 1.9. Let $G(P)$ be the graph with vertex set $\Lambda$ and edge set

$$
\left\{\left(s, s^{\prime}\right): s<_{P} s^{\prime} \text { and } \nexists s^{\prime \prime} \in S \text { s.t. } s<_{P} s^{\prime \prime}<_{P} s^{\prime}\right\}
$$

where $s<_{P} s^{\prime}$ iff $s<s^{\prime}$ and $s$ and $s^{\prime}$ are in the same block of $P$.

A bump diagram of $P$ is a drawing of $G(P)$ in the plane in which the elements of $\Lambda$ are arrayed along a horizontal line in their given order, all edges lie above this line, and no two edges intersect more than once.

Definition 1.10. $P$ is noncrossing if its bump diagram contains no two crossing edges, equivalently if $G(P)$ contains no two edges of form $(a, c),(b, d)$ with $a<b<$ $c<d$.

Definition 1.11. $P$ is nonnesting if its bump diagram contains no two nested edges, equivalently if $G(P)$ contains no two edges of form $(a, d),(b, c)$ with $a<b<c<d$.

The words "noncrossing" and "nonnesting" perhaps properly belong as predicates to the bump diagram of $P$ and not to $P$ itself, but we will mostly abuse the terminology slightly and use them as just defined. We will denote the set of classical noncrossing and nonnesting partitions for $W$ by $N C^{\mathrm{cl}}(W)$, resp. $N N^{\mathrm{cl}}(W)$. To define these sets it remains only to specify the ordered ground set.

For $N N^{\text {cl }}(W)$, the ordering we use is read off the line of integers in Figure 1.

Definition 1.12. A classical nonnesting partition for a classical reflection group $W$ is a classical partition for $W$ nonnesting with respect to the ground set

$$
\begin{array}{cl}
1<\cdots<n & \text { if } W=A_{n-1} \\
-n<\cdots<-1<0<1<\cdots<n & \text { if } W=B_{n} \\
-n<\cdots<-1<1<\cdots<n & \text { if } W=C_{n} \\
-n<\cdots<-1,1<\cdots<n & \text { if } W=D_{n} .
\end{array}
$$

A few remarks on the interpretation of these are in order.

Classical nonnesting partitions for $B_{n}$ differ from those for $C_{n}$, reflecting the different root posets. We have specified that 0 is part of the ordered ground set for $B_{n}$. Despite that, per Definition 1.7. 0 can't occur in a classical partition, it is harmless to consider it present, coming from the zero vector and forming part of (or perhaps all of) the zero block. Its presence is quite necessary when drawing bump diagrams: the dot 0 "ties down" a problematic edge of the zero block in the middle, preventing it from nesting with the others.

The ground set for classical nonnesting partitions for $D_{n}$ is not totally ordered but is merely a strict weak ordering, in which 1 and -1 are incomparable. Definitions 1.9 and 1.11 generalise cleanly to this situation, with no amendments to the text of the definitions themselves. That is, in a classical nonnesting partition for $D_{n}$, an edge with 1 as vertex and another with -1 as vertex are never considered to nest. We diverge in purely cosmetic fashion from Athanasiadis and reinforce this last point by aligning these two dots vertically when drawing a type $D$ nonnesting bump diagram.

Figure 3 exemplifies Definition 1.12, giving one nonnesting bump diagram for each classical type.

For $N C^{\mathrm{cl}}(W)$, the ordering we use is read off of the standard Coxeter elements in 1.3 . 

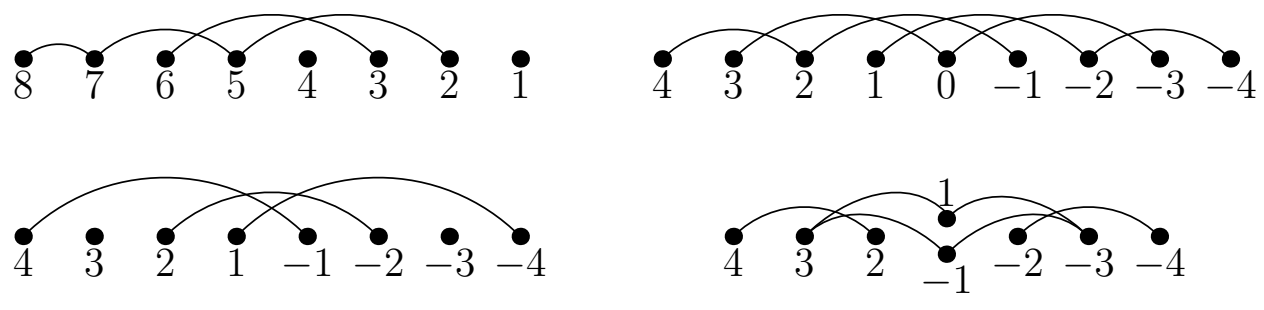

Figure 3. Examples of nonnesting bump diagrams in (top) $A_{7}$, $B_{4}$; (bottom) $C_{4}, D_{4}$.

Definition 1.13. A classical noncrossing partition for a classical reflection group $W$ not of type $D$ is a classical partition for $W$ noncrossing with respect to the ground set

$$
\begin{array}{cl}
1<\cdots<n & \text { if } W=A_{n-1} \\
-1<\cdots<-n<1<\cdots<n & \text { if } W=B_{n} \\
-1<\cdots<-n<1<\cdots<n & \text { if } W=C_{n} .
\end{array}
$$

Observe that the order $<$ in these ground sets differs from those for nonnesting partitions.

For $D_{n}$ the standard Coxeter element is not a cycle, so we can't carry this through, though it's not too far from true that the ground set is $-2<\cdots<-n<$ $2<\cdots<n$. We return to type $D$ shortly.

These orderings come from cycles, so as one might expect, if $P$ is noncrossing with respect to $(\Lambda,<)$, it's also noncrossing with respect to any rotation $\left(\Lambda,<^{\prime}\right)$ of $(\Lambda,<)$, i.e. any order $<^{\prime}$ on $\Lambda$ given by

$$
s<t \quad \Leftrightarrow \quad t \leq s_{0}<s \text { or } s<t \leq s_{0} \text { or } s_{0}<s<t
$$

for some $s_{0} \in \Lambda$ fixed. Reflecting this, given any classical partition $P$, we may bend round the line on which the vertices of a bump diagram for $P$ lie into a circle, and if we like supply extra edges for newly adjacent members of the same block, obtaining a circular bump diagram. Then $P$ will be noncrossing if and only if, for every pair of distinct blocks $B, B^{\prime}$ of $P$, the convex hulls of the dots representing $B$ and $B^{\prime}$ are disjoint. For example, Figure 4 is the type $B$ or $C$ noncrossing partition of Figure 2 rendered circularly.

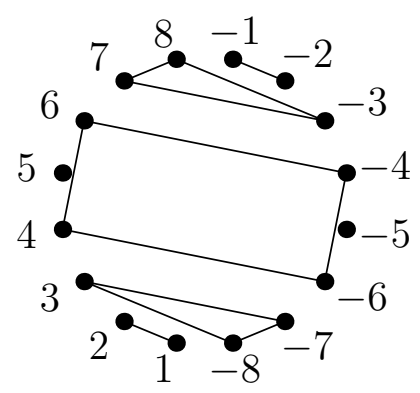

Figure 4. The partition of Figure 2 rendered circularly.

The subtleties that occur defining classical noncrossing partitions in type $D$ are significant, and historically it proved troublesome to provide the correct notion 
for this case. Reiner's first definition [15] of classical noncrossing partitions for type $D$ was later superceded by that of Bessis and Brady and Watt [5], 7] and Athanasiadis and Reiner [4, which we use here, for its better agreement with the uniform definition of $N C\left(D_{n}\right)$. Indeed definitions 1.9 through 1.11 require tweaking to handle type $D$ adequately. (This said we'll still use the name "bump diagram" for a diagram of a classical noncrossing partition for $D_{n}$.)

Definition 1.14. A classical noncrossing partition $\pi$ for $D_{n}$ is a classical partition for $D_{n}$ such that there exists $c \in\{2, \ldots, n\}$ for which $\pi$ is noncrossing with respect to both of the ordered ground sets

$$
-2<\cdots<-c<-1<-(c+1)<\cdots<-n<2<\cdots<c<1<c+1<\cdots<n
$$

and

$$
-2<\cdots<-c<1<-(c+1)<\cdots<-n<2<\cdots<c<-1<c+1<\cdots<n
$$

The set of these will be denoted $N C^{\mathrm{cl}}\left(D_{n}\right)$.

We will draw these circularly. Arrange dots labelled $-2, \ldots,-n, 2, \ldots, n$ in a circle and place 1 and -1 in the middle. We let 1 and -1 be drawn coincidently, after [4, although it would be better to use two circles as in [11, with a smaller one in the center on which only 1 and -1 lie. Then a $D_{n}$ partition $\pi$ is noncrossing if and only if no two blocks in this circular bump diagram have intersecting convex hulls, except possibly two blocks $\pm B$ meeting only at the middle point. The edges we will supply in these circular diagrams are those delimiting the convex hulls of the blocks. See Figure 5 for an example.

Note that a zero block of precisely two elements cannot occur in a classical partition for $D_{n}$ : a singular equation $x_{i}=0$ cannot arise describing a subspace of $\Pi\left(D_{n}\right)$ which is the intersection of hyperplanes of form $x_{i}= \pm x_{j}$. So the two central dots \pm 1 belong to different blocks unless they are both inside the convex hull of some set of vertices among $\pm\{2, \ldots, n\}$ which are part of the sole zero block.

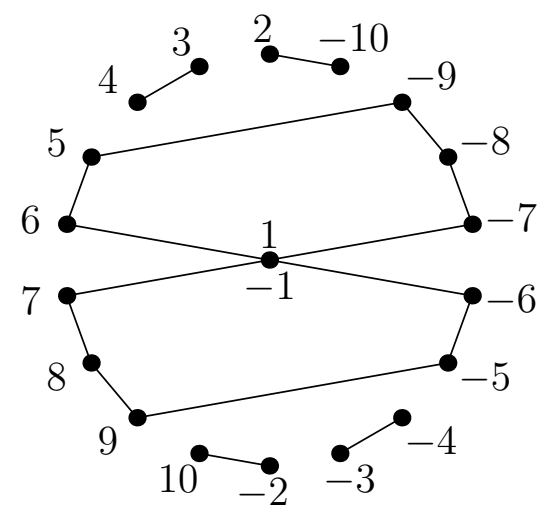

Figure 5. Example of a circular bump diagram for a type $D$ classical nonnesting partition.

We state without proof the relations between these classical noncrossing and nonnesting partitions and the uniform ones. For $w \in W$, let the fixed space $\operatorname{Fix}(w)$ of $w$ be the subspace of $V(W)$ consisting of vectors fixed by $w$, i.e. Fix $(w)=$ $\operatorname{ker}(w-1)$. 
Proposition 1.15. The map $f_{N C}: w \mapsto \operatorname{Part}(\operatorname{Fix}(w))$ is a bijection between $N C(W, c)$ and $N C^{\mathrm{cl}}(W)$, where $c$ is the element in (1.3). Moreover it is an isomorphism of posets, where $N C(W, c)$ is given the absolute order and $N C^{\mathrm{cl}}(W)$ the reverse refinement order.

Proposition 1.16. The map $f_{N N}: S \mapsto \operatorname{Part}\left(\bigcap_{\alpha \in S} \alpha^{\perp}\right)$ is a bijection between $N N(W)$ and $N N^{\mathrm{cl}}(W)$.

This yields the following elementary descriptions of how to obtain the edges in a bump diagram. Starting from an antichain $\pi \in N N(W)$, each root gives an edge of the nonnesting bump diagram (and its negative), between the two integers in line with it per the discussion before Figure 1. Starting from a group element $\pi \in N C(W)$, each orbit of the action of $\pi$ on $\left\{ \pm e_{i}: i=1, \ldots, n\right\} \cup\{0\}$ gives a block of the noncrossing bump diagram, with an edge between each element and its image under the permutation representation.

Proposition 1.17. Consider a reduced expression in $T$ for some $w \in W$ where $W$ is a Weyl group,

$$
w=t_{\alpha_{1}} t_{\alpha_{2}} t_{\alpha_{3}} \ldots t_{\alpha_{m}} \text { and } \alpha_{1}, \alpha_{2}, \ldots, \alpha_{m} \in \Phi
$$

Then $\operatorname{Fix}(w)=\bigcap_{i=1}^{m} \alpha_{i}^{\perp}$.

The $\supseteq$ containment clearly holds. Them the proposition is an immediate consequence of [8, Lemma 2], which tells us that the two spaces have the same dimension,

Corollary 1.18. Let $\rho$ be a permutation of the set $[m]$. Define

$$
w_{\rho}=t_{\alpha_{\rho(1)}} t_{\alpha_{\rho(2)}} t_{\alpha_{\rho(3)}} \ldots t_{\alpha_{\rho(m)}} .
$$

Then $\operatorname{Fix}(w)=\operatorname{Fix}\left(w_{\rho}\right)$.

So, if we are given an antichain $A$ of the root poset for some group $W$, we may define $\operatorname{Fix}(A)$ to be $\operatorname{Fix}\left(\pi_{A}\right)$ where $\pi_{A}$ is the product of the elements of $A$ in any order. The elements of an antichain are linearly independent so Corollary 1.18 shows that $\operatorname{Fix}(A)$ is well-defined. See [16].

Lastly, the distribution of classical noncrossing and nonnesting partitions with respect to type is well-behaved. In the noncrossing case, the images of the conjugacy classes of the group $W$ itself are the same as these conjugacy classes of the action of $W$ on $\Pi(W)$.

One can check that

Proposition 1.19. Two subspaces $L, L^{\prime} \in \Pi(W)$ are conjugate if and only if both of the following hold:

- the multisets of block sizes $\{|C|: C \in \operatorname{Part}(L)\}$ and $\left\{|C|: C \in \operatorname{Part}\left(L^{\prime}\right)\right\}$ are equal;

- if either $\operatorname{Part}(L)$ or $\operatorname{Part}\left(L^{\prime}\right)$ has a zero block, then both do, and these zero blocks have equal size.

For example, the type $A$ specialisation of this result, where zero blocks are irrelevant and we drop the redundant negative elements, says that the conjugacy classes of the symmetric group $A_{n-1}$ on $n$ elements are enumerated by the partitions of the integer $n$.

We close this section with the statement of the equidistribution result of Athanasiadis and Reiner [4]. 
Theorem 1.20. Let $W$ be a Weyl group. Let $f_{N C}$ and $f_{N N}$ be the functions of Propositions 1.15 and 1.16 . For any type $\lambda$ we have

$$
\mid\left(\text { type } \circ f_{N C}\right)^{-1}(\lambda)|=|\left(\text { type } \circ f_{N N}\right)^{-1}(\lambda) \mid \text {. }
$$

\section{A tYPE-PRESERVING BIJECTION FOR CLASSICAL GROUPS}

Throughout this section $W$ will be a classical reflection group. Partitions will be drawn and spoken of with the greatest elements of their ground sets to the left.

Given any partition, define the order $<_{\operatorname{lp}}$ on those of its blocks containing positive elements so that $B<_{\operatorname{lp}} B^{\prime}$ if and only if the least positive element of $B$ is less than the least positive element of $B^{\prime}$.

The notation $N C(W)$ with the Coxeter element omitted will mean $N C(W, c), c$ being the element in 1.3 .

By convention, when we define partition statistics, we shall observe the convention that Roman letters (like $a$ ) denote ground set elements or tuples thereof, and Greek letters (like $\mu$ ) denote cardinalities or tuples thereof.

\subsection{Statement of the central theorem. We establish some notation.}

Definition 2.1. Let $\Psi^{n}$ be the set of $n$-tuples with entries in $\{1,0,-1\}$. For any $u \in \Psi^{n}$ define $\#(u, 1)$ to be the number of entries equal to 1 in $u$ and define $\#(u,-1)$ analagously. Let $<_{\text {lex }}$ be the lexicographic order on $n$-tuples. For any two vectors $a, b \in \mathbb{Z}^{n}$, let $\underline{a}$ be the set of elements of $\mathbb{Z}^{n}<_{\text {lex }}$-less than or equal to $a$ and let $\|a-b\|=\left(\left|a_{1}-b_{1}\right|, \ldots,\left|a_{n}-b_{n}\right|\right)$.

To any nonnesting or noncrossing partition $x$ of $W$ we associate a set $\Omega_{x}$ which is constructed inductively with $i$ increasing from 1 to $n$ stepwise. Initially, we begin with $\Omega_{x}=\emptyset$. In step $i$, let $u_{i}$ be the element of $\Psi^{n} \cap \operatorname{Fix}(x)$ with $\left\|e_{i}-u_{i}\right\|<_{\text {lex }^{-}}$ minimal (actually $\left\|e_{i}-u_{i}\right\| \in \Psi^{n}$ ). Whenever $u_{i}$ is linearly independent with the elements of $\Omega_{x}$, let $\Omega_{x}=\Omega_{x} \cup\left\{-u_{i}\right\}$ if $u_{i}$ has some entry -1 and let $\Omega_{x}=\Omega_{x} \cup\left\{u_{i}\right\}$ if not. Let $\Gamma_{x}$ be the number of canonical coordinate projections of $\operatorname{Fix}(x)$ with trivial image $\{0\}$.

Lastly, let $E$ be the canonical basis of $\mathbb{R}^{n}$.

Theorem 2.2. Let $x \in N N(W)$ [resp. $x \in N C(W)$ ]. Then, there is a unique $y \in N C(W)$ [resp. $y \in N N(W)$ ] for which $\Gamma_{x}=\Gamma_{y}$ and such that the sets $\Omega_{x}$ and $\Omega_{y}$ are related to each other in the following way:

There is a bijection $\sigma$ between $\Omega_{x}$ and $\Omega_{y}$ such that for each $u \in \Omega_{x}$ we have $\sigma(u) \in \Omega_{y}$ satisfying

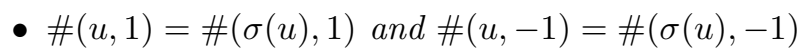

- $|\underline{u} \cap E|=|\sigma(u) \cap E|$

- $\left|\underline{u} \cap \Omega_{x}\right|=\overline{\mid \sigma(u)} \cap \Omega_{y} \mid$

- the product of the first two nonzero components of $u$ and $\sigma(u)$ is not equal whenever $\#(u,-1)>1$ and $\#(u, 1)>0$

Consequently, the induced mapping establishes a bijection between noncrossing and nonnesting partitions preserving orbital type. 
Example 2.3. Let $x$ be the nonnesting partition $\left\{e_{2}+e_{1}, e_{5}-e_{1}, e_{6}-e_{2}, e_{8}-\right.$ $\left.e_{6}, e_{7}-e_{3}\right\}$ of the group $C_{8}$. The fixed space $\operatorname{Fix}(x)$ is the following intersection in $\mathbb{R}^{8}$ :

$$
\left\{v \mid v_{1}=-v_{2}\right\} \cap\left\{v \mid v_{1}=v_{5}\right\} \cap\left\{v \mid v_{2}=v_{6}\right\} \cap\left\{v \mid v_{6}=v_{8}\right\} \cap\left\{v \mid v_{3}=v_{7}\right\}
$$

This is the set $\left\{v \in \mathbb{R}^{8} \mid v_{1}=v_{5}=-v_{2}=-v_{6}=-v_{8}\right.$ and $\left.v_{3}=v_{7}\right\}$. We can see $\Gamma_{x}=0$ and also

$$
\Omega_{x}=\{(-1,1,0,0,-1,1,0,1),(0,0,1,0,0,0,1,0),(0,0,0,1,0,0,0,0)\}
$$

Now, we may check

$$
\begin{aligned}
& t_{e_{7}-e_{6}} t_{e_{8}-e_{7}} t_{e_{1}+e_{8}} t_{e_{2}-e_{1}} t_{e_{5}-e_{3}} t_{e_{5}-e_{2}} t_{e_{4}-e_{2}} t_{2 e_{5}} \\
& \quad=t_{2 e_{1}} t_{e_{2}-e_{1}} t_{e_{3}-e_{2}} t_{e_{4}-e_{3}} t_{e_{5}-e_{4}} t_{e_{6}-e_{5}} t_{e_{7}-e_{6}} t_{e_{8}-e_{7}}
\end{aligned}
$$

so $y=t_{e_{7}-e_{6}} t_{e_{8}-e_{7}} t_{e_{1}+e_{8}} t_{e_{2}-e_{1}} t_{e_{5}-e_{3}}$ is less than a Coxeter element in the absolute order and thus is a noncrossing partition of $C_{8}$. We can calculate $\operatorname{Fix}(y)=\{v \in$ $\mathbb{R}^{8} \mid v_{1}=v_{2}=-v_{6}=-v_{7}=-v_{8}$ and $\left.v_{3}=v_{5}\right\}$, so $\Gamma_{y}=0$ and also

$$
\Omega_{y}=\{(-1,-1,0,0,0,1,1,1),(0,0,1,0,1,0,0,0),(0,0,0,1,0,0,0,0)\}
$$

Finally, let $\sigma: \Omega_{x} \mapsto \Omega_{y}$ be given by the assignments in the left column of the following table.

\begin{tabular}{r|c|c|c|c} 
& $\#(u, 1)$ & $\#(u,-1)$ & $|\underline{u} \cap E|$ & $\left|\underline{u} \cap \Omega_{x}\right|$ \\
\hline $\begin{array}{r}(-1,1,0,0,-1,1,0,1) \\
(-1,-1,0,0,0,1,1,1)\end{array}$ & 3 & 2 & 0 & 1 \\
\hline $\begin{array}{r}(0,0,1,0,0,0,1,0) \\
\mapsto(0,0,1,0,1,0,0,0)\end{array}$ & 2 & 0 & 6 & 3 \\
\hline $\begin{array}{r}(0,0,0,1,0,0,0,0) \\
(0,0,0,1,0,0,0,0)\end{array}$ & 1 & 0 & 5 & 2 \\
\hline
\end{tabular}

The remaining columns record the values in Theorem 2.2 in each case they are equally true of $u$ (and $\Omega_{x}$ ) and of $\sigma(u)$ (and $\Omega_{y}$ ). The last bullet in the Theorem only has force in the first line, where it also holds. So $\sigma$ satisfies the required properties.

In the remainder of the paper we will prove Theorem 2.2. The four sections that follow will give, in a case by case fashion, the individual type preserving bijections for each of the classical types that arise from the theorem. Then in 2.6 we tie these together and complete the proof.

2.2. Type $A$. The bijection in type $A$, which forms the foundation of the ones for the other types, is due to Athanasiadis [3, §3]. We include it here to make this foundation explicit and to have bijections for all the classical groups in one place.

Let $\pi$ be a classical partition for $A_{n-1}$. Let $M_{1}<_{\mathrm{lp}} \cdots<_{\mathrm{lp}} M_{m}$ be the blocks of $\pi$, and $a_{i}$ the least element of $M_{i}$, so that $a_{1}<\cdots<a_{m}$. Let $\mu_{i}$ be the cardinality of $M_{i}$. Define the two statistics $a(\pi)=\left(a_{1}, \ldots, a_{m}\right)$ and $\mu(\pi)=\left(\mu_{1}, \ldots, \mu_{m}\right)$.

It turns out that classical nonnesting and noncrossing partitions are equidistributed with respect to these partition statistics, and that they uniquely determine one partition of either kind. This will be the mode in which we present all of our bijections, which will differ from this one in the introduction of more statistics.

We will say that a list of partition statistics $S$ establishes a bijection for a classical reflection group $W$ if, given either a classical noncrossing partition $\pi^{\mathrm{NC}}$ or a classical 
nonnesting partition $\pi^{\mathrm{NN}}$ for $W$, the other one exists uniquely such that $s\left(\pi^{\mathrm{NC}}\right)=$ $s\left(\pi^{\mathrm{NN}}\right)$ for all $s \in S$. We will say it establishes a type-preserving bijection if furthermore $\pi^{\mathrm{NC}}$ and $\pi^{\mathrm{NN}}$ always have the same type.

Theorem 2.4. The statistics $(a, \mu)$ establish a type-preserving bijection for $A_{n-1}$.

The type-preserving assertion in Theorem 2.4 is easy: by Proposition 1.19 the tuple $\mu$ determines the type of any partition that yields it. As for the bijection itself, we will sketch two different descriptions of the process for converting back and forth between classical noncrossing and nonnesting partitions with the same tuples $a, \mu$, with the intent that they will provide the reader with complementary suites of intuition.

To give an example, Figures 6 and 7 show step by step the operation of this bijection in each direction, in the chain-by-chain fashion of our first proof. In these figures, the chain $M_{i}$ being considered appears in bold. In the partitions being constructed, the elements which are shown with labels and thick dots are those less than or equal to the least element $a_{i}$ of the last placed chain $M_{i}$. As we'll see in the proof, no subsequently placed chain can include an element less than $a_{i}$, so these labels are correct.
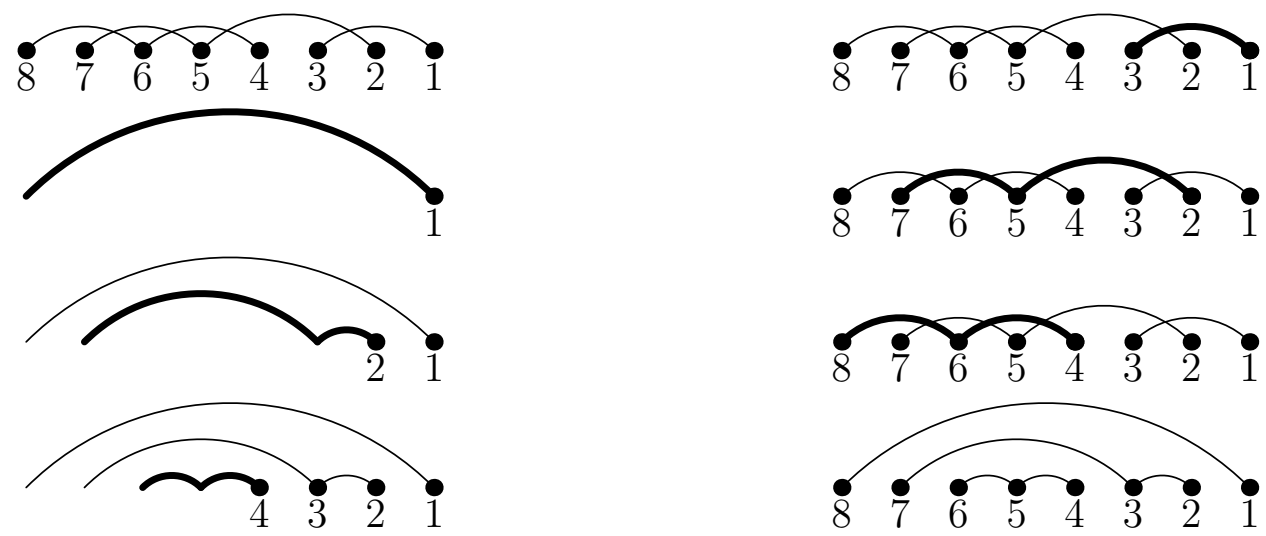

Figure 6 . The bijection of type $A$ running chain by chain (from left to right, top to bottom) converting a nonnesting partition to a noncrossing one. The partitions correspond to $a=(1,2,4), \mu=$ $(2,3,3)$.

Proof of Theorem 2.4: chain by chain. By a chain we will mean a sort of incompletely specified block of a classical partition, or a connected component of a bump diagram: a chain has a definite cardinality (or length) but may have unknown elements. The lengths of the chains of $\pi$ are determined by $\mu(\pi)$. We can view the chains as abstract unlabelled graphs in the plane, and our task is that of labelling and thereby positioning the vertices of these chains in such a way that the result is nonnesting or noncrossing, as desired.

To compute the bijection we will inductively place the chains $M_{1}, \ldots, M_{m}$, in that order. When we say a set $\mathcal{M}$ of chains is placed, we mean that all pairwise order relations between the elements of the chains in $\mathcal{M}$ are known. The effect is 

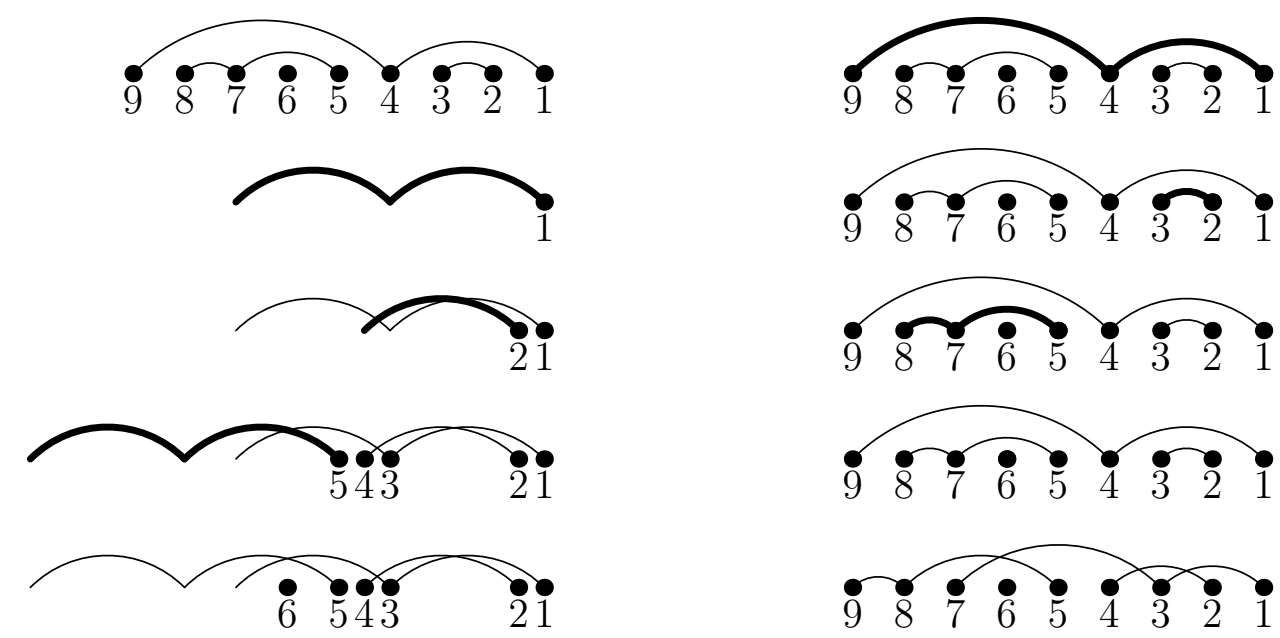

Figure 7 . The bijection of type $A$ running chain by chain (from left to right, top to bottom) converting a noncrossing partition to a nonnesting one. The partitions correspond to $a=(1,2,5,6)$, $\mu=(3,2,3,1)$.

that if $\mathcal{M}$ is placed, we can draw the chains of $\mathcal{M}$ in such a way that the bump diagram of any classical partition $\pi$ containing blocks whose elements have the order relations of $\mathcal{M}$ can be obtained by drawing additional vertices and edges in the bump diagram, without redrawing the placed chains.

Suppose we start with $\pi^{\mathrm{NN}}$ and want to build the noncrossing diagram of $\pi^{\mathrm{NC}}$. Suppose that, for some $j \leq n$, we have placed $M_{i}$ for all $i<j$. To place $M_{j}$, we specify that its least element is to be the $a_{j}$ th least element among the elements of all of $M_{1}, \ldots, M_{j-1}, M_{j}$, and that its remaining elements are to be ordered in the unique possible way so that the placed chains form no crossing. In this instance, this means that all the elements of $M_{j}$ should be placed consecutively, in immediate succession, as in Figure 6 .

To build $\pi^{\mathrm{NC}}$ from $\pi^{\mathrm{NN}}$ the procedure is the same, except that we must order the elements of $M_{j}$ in the unique possible way so that the placed chains form no nesting. Concretely, these order relations are the ones we get if every edge is drawn with its vertices the same distance apart on the line they lie on, as in Figure 7.

Note that, in both directions, all the choices we made were unique, so the resulting partitions are unique.

We remark that viewing each block of $\pi^{\mathrm{NN}}$ as a chain with a fixed spacing is a particularly useful picture in terms of the connection between nonnesting partitions and chambers of the Shi arrangement [3, §5].

Proof of Theorem 2.4: dot by dot. Let $M_{1}, \ldots, M_{m}$ be the blocks of a classical nonnesting partition $\pi^{\mathrm{NN}}$, such that the least vertex of $M_{i}$ is $a_{i}$. We describe an algorithm to build up a classical noncrossing partition $\pi^{\mathrm{NC}}$ with the same tuples $a$ and $\mu$ by assigning the elements $1, \ldots, n$, in that order, to blocks.

The algorithm maintains a set $\mathcal{O}$ of open blocks: an open block is a pair $(C, \kappa)$ where $C$ is a subset of the ground set of $\pi^{\mathrm{NN}}$ and $\kappa$ a nonnegative integer. We think 
of $C$ as a partially completed block of $\pi^{\mathrm{NC}}$ and $\kappa$ as the number of elements which must be added to $C$ to complete it. If $\mathcal{O}$ ever comes to contain an open block of form $(C, 0)$, we immediately drop this, for it represents a complete block. When we begin constructing $\pi^{\mathrm{NC}}$, the set $\mathcal{O}$ will be empty.

Suppose we've assigned the elements $1, \ldots, j-1$ to blocks of $\pi^{\mathrm{NC}}$ already, and want to assign $j$. If $j$ occurs as one of the $a_{i}$, then we add a new singleton block $\{j\}$ to $\pi^{\mathrm{NC}}$ and add $\left(\left\{a_{i}\right\}, \mu_{i}-1\right)$ to $\mathcal{O}$. Otherwise, we choose an open block from $\mathcal{O}$ according to the

Noncrossing open block policy. Given $\mathcal{O}$, choose from it the open block $(C, \kappa)$ such that the maximum element of $C$ is maximal.

We add $j$ to this open block, i.e. we replace the block $C$ of $\pi^{\mathrm{NC}}$ by $C^{\prime}:=C \cup\{j\}$ and replace $(C, \kappa)$ by $\left(C^{\prime}, \kappa-1\right)$ within $\mathcal{O}$. The desired partition $\pi^{\mathrm{NC}}$ is obtained after assigning all dots.

The central observation to make is that this policy indeed makes $\pi^{\mathrm{NC}}$ noncrossing, and there's a unique way to follow it. Making a crossing of two edges $(a, c)$ and $(b, d)$, where $a<b<c<d$, requires assigning $c$ to an open block whose greatest element is then $a$, when there also exists one with greatest element $b>a$, which is witnessed to have been open at the time by its later acquisition of $d$; this is in contravention of the policy.

To recover $\pi^{\mathrm{NN}}$ uniquely from $\pi^{\mathrm{NC}}$, the same algorithm works, with one modification: instead of the noncrossing open block policy we use the

Nonnesting open block policy. Given $\mathcal{O}$, choose from it the open block $(C, \kappa)$ such that the maximum element of $C$ is minimal.

This policy makes $\pi^{\mathrm{NN}}$ nonnesting and unique for a similar reason. If there are nested edges $(a, d)$ and $(b, c)$, where $a<b<c<d$, then $c$ was added to the block containing $b$ when by policy it should have gone with $a$, which was in an open block.

A careful study of either of these proofs provides a useful characterisation of the pairs of tuples $a, \mu$ that are the statistics of a classical nonnesting or noncrossing partition of type $A$.

Corollary 2.5. Suppose we are given a pair of tuples of positive integers $a=$ $\left(a_{1}, \ldots, a_{m_{1}}\right), \mu=\left(\mu_{1}, \ldots, \mu_{m_{2}}\right)$ and let $n>0$. Define $a_{0}=0$ and $\mu_{0}=1$. Then, $a$ and $\mu$ represent a classical noncrossing or nonnesting partition for $A_{n-1}$ if and only if

(1) $m_{1}=m_{2}=m$;

(2) $n=\sum_{k=1}^{m} \mu_{k} ;$ and

(3) $a_{i-1}<a_{i} \leq \sum_{k=0}^{i-1} \mu_{k}$ for $i=1,2, \ldots, m$.

2.3. Type $C$. In the classical reflection groups other than $A_{n}$, the negative elements of the ground set must be treated, and so it will be useful to have some terminology to deal with these.

Definition 2.6. A positive block of a classical partition $\pi$ is a block of $\pi$ that contains some positive integer; similarly a negative block contains a negative integer. A switching block of $\pi$ is a block of $\pi$ that contains both positive and nonpositive 
elements, and a nonswitching block is one that contains only positive elements or only nonpositive elements.

A single edge of the bump diagram is positive or negative or switching or nonswitching if it would have those properties as a block of size 2 .

Let $\pi$ be a classical partition for $C_{n}$. Given $\pi$, let $M_{1}<_{\text {lp }} \cdots<_{\text {lp }} M_{m}$ be the positive nonswitching blocks of $\pi$, and $a_{i}$ the least element of $M_{i}$. Let $\mu_{i}$ be the cardinality of $M_{i}$. These two tuples are reminiscent of type $A$. Let $P_{1}<_{\text {lp }}$ $\cdots<_{\text {lp }} P_{k}$ be the switching blocks of $\pi$, let $p_{i}$ be the least positive element of $P_{i}$, and let $\nu_{i}$ be the number of positive elements of $P_{i}$. Define the three statistics $a(\pi)=\left(a_{1}, \ldots, a_{m}\right), \mu(\pi)=\left(\mu_{1}, \ldots, \mu_{m}\right), \nu(\pi)=\left(\nu_{1}, \ldots, \nu_{k}\right)$. We have

$$
n=\sum_{i=1}^{m} \mu_{i}+\sum_{j=1}^{k} \nu_{j} .
$$

Theorem 2.7. The statistics $(a, \mu, \nu)$ establish a type-preserving bijection for $C_{n}$.

Figure 8 illustrates a pair of partitions related under the resulting bijection.

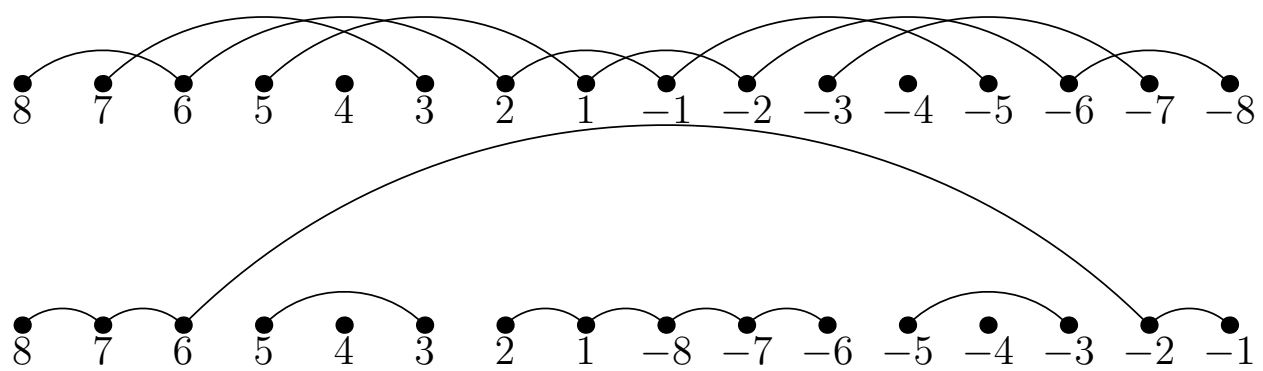

Figure 8 . The type $C$ nonnesting (top) and noncrossing (bottom) partitions corresponding to $a=(3,4), \mu=(2,1), \nu=(2,3)$.

Proof. We state a procedure for converting back and forth between classical noncrossing and nonnesting partitions that preserve the values $a, \mu$, and $\nu$. Suppose we start with a partition $\pi$, be it noncrossing $\pi^{\mathrm{NN}}$ or nonnesting $\pi^{\mathrm{NC}}$, so that we want to find the partition $\pi^{\prime}$, being $\pi^{\mathrm{NC}}$ or $\pi^{\mathrm{NN}}$ respectively. From $a, \mu$, and $\nu$ we inductively construct the positive side of $\pi^{\prime}$, that is the partition it induces on the set of positive indices $[n]$, which will determine $\pi^{\prime}$ by invariance under negation.

First we describe it from the chain-by-chain viewpoint. In the bump diagram of $\pi$, consider the labelled connected component representing $P_{i}$, which we call the chain $P_{i}$. Let the (unlabelled) partial chain $P_{i}^{\prime}$ be the abstract unlabelled connected graph obtained from the chain $P_{i}$ by removing its nonpositive nonswitching edges and nonpositive vertices, leaving the unique switching edge incomplete, i.e. drawn as a partial edge with just one incident vertex, and by dropping the labels. Notice how the tuple $\nu$ allows us to draw these partial chains. The procedure we followed for type $A$ will generalise to this case, treating the positive parts of the switching edges first.

We want to obtain the bump diagram for $\pi^{\prime}$, so we begin by using $\nu$ to partially draw the chains representing its switching blocks: we draw only the positive edges 
(switching and nonswitching) of every chain, leaving the unique switching edge incomplete. This is done by reading $\nu$ from back to front and inserting, each partial switching chain $P_{i}^{\prime}$ in turn with its rightmost dot placed to the right of all existing chains, analogously to type $A$. In the noncrossing case, we end up with every vertex of $P_{i}^{\prime}$ being strictly to the right of every vertex of $P_{j}^{\prime}$ for $i<j$. In the nonnesting case, the vertices of the switching edges will be exactly the $k$ first positions from right to left among all the vertices of $P_{1}^{\prime}, \ldots, P_{k}^{\prime}$. It remains to place the nonswitching chains $M_{1}, M_{2}, \ldots, M_{m}$, and this we do also as in the type $A$ bijection, except that at each step, we place the rightmost vertex of $M_{j}$ so as to become the $a_{j}$ th vertex, counting from right to left, relative to the chains $M_{j-1}, \ldots, M_{1}$ and the partial chains $P_{1}^{\prime}, P_{2}^{\prime}, \ldots, P_{k}^{\prime}$ already placed.

To take the dot-by-dot viewpoint, the type $A$ algorithm can be used with only one modification, namely that $\mathcal{O}$ begins nonempty. It is initialised from $\nu$, as

$$
\mathcal{O}=\left\{\left(\left\{P_{i}^{-}\right\}, \nu_{i}\right): i=1, \ldots, k\right\},
$$

where $P_{i}^{-}$is a fictive element that represents the negative elements of $P_{i}$ which are yet to be added. We must also specify how these fictive elements compare, for use in the open block policies. A fictive element is always less than a real element. In the noncrossing case $P_{i}^{-}>P_{j}^{-}$iff $i<j$, whereas in the nonnesting case $P_{i}^{-}>P_{j}^{-}$ iff $i>j$; the variation assures that $P_{1}^{-}$is chosen first in either case.

Now we have the positive side of $\pi^{\prime}$. We copy these blocks down again with all parts negated, and end up with a set of incomplete switching blocks $P_{1}^{*}, \ldots, P_{k}^{*}$ on the positive side and another equinumerous set $-P_{1}^{*}, \ldots,-P_{k}^{*}$ on the negative side that we need to pair up and connect with edges in the bump diagram.

There is a unique way to connect these incomplete blocks to get the partition $\pi^{\prime}$, be it $\pi^{\mathrm{NC}}$ or $\pi^{\mathrm{NN}}$. In every case $P_{i}^{*}$ gets connected with $-P_{k+1-i}^{*}$, and in particular symmetry under negation is attained. If there is a zero block it arises from $P_{(k+1) / 2}^{*}$.

Finally, $\pi$ and $\pi^{\prime}$ have the same type. Since the $P_{i}^{*}$ are paired up the same way in each, including any zero block, $\mu$ and $\nu$ determine the multiset of block sizes of $\pi$ and $\pi^{\prime}$ and the size of any zero block, in identical fashion in either case. Then this is Proposition 1.19 .

Again, a careful look at the preceding proof gives the characterization of the tuples that describe classical noncrossing and nonnesting partitions for type $C$.

Corollary 2.8. Suppose we are given some tuples of positive integers $a=\left(a_{1}, \ldots, a_{m_{1}}\right)$, $\mu=\left(\mu_{1}, \ldots, \mu_{m_{2}}\right), \nu=\left(\nu_{1}, \ldots, \nu_{k}\right)$ and let $n>0$. Define $a_{0}=0$ and $\mu_{0}=1$. Then, $a, \mu$ and $\nu$ represent a classical noncrossing or nonnesting partition for $C_{n}$ if and only if

(1) $m_{1}=m_{2}=m$;

(2) $n=\sum_{i=1}^{m} \mu_{i}+\sum_{j=1}^{k} \nu_{j}$;

(3) $a_{i-1}<a_{i} \leq \sum_{k=0}^{i-1} \mu_{k}+\sum_{j=1}^{k} \nu_{j}$ for $i=1,2, \ldots, m$.

2.4. Type $B$. We will readily be able to modify our type $C$ bijection to handle type $B$. Indeed, if it weren't for our concern about type in the sense of Definition 1.8 . we would already possess a bijection for type $B$, differing from the type $C$ bijection only in pairing up the incomplete switching blocks in a way respecting the presence 
of the element 0 . Our task is thus to adjust that bijection to recover the typepreservation.

If $\pi$ is a classical partition for $B_{n}$, we define the tuples $a(\pi), \mu(\pi)$ and $\nu(\pi)$ as in type $C$.

Notice that classical noncrossing partitions for $B_{n}$ and for $C_{n}$ are identical, and that the strictly positive part of any classical nonnesting partition for $B_{n}$ is also the strictly positive part of some nonnesting $C_{n}$-partition, though not necessarily one of the same type. Thus Corollary 2.8 characterises the classical noncrossing or nonnesting partitions for $B_{n}$ just as well as for $C_{n}$.

Suppose $\pi$ is a classical nonnesting partition for $B_{n}$. In two circumstances its tuples $a(\pi), \mu(\pi), \nu(\pi)$ also describe a unique nonnesting partition for $C_{n}$ of the same type: to be explicit, this is when $\pi$ does not contain a zero block, and when the unique switching chain in $\pi$ is the one representing the zero block. If $P_{1}<_{\text {lp }}$ $\cdots<_{\text {lp }} P_{k}$ are the switching blocks of $\pi$, then $\pi$ contains a zero block and more than one switching chain if and only if $k$ is odd and $k>1$. We notice that $P_{k}$ must be the zero block. On the other hand, if $\pi^{\mathrm{C}}$ is a classical nonnesting partition for $C_{n}$, the zero block must be $P_{(k+1) / 2}$. Reflecting this, our bijection will be forced to reorder $\nu$ to achieve type preservation.

Generalising our prior machinery, we will say that two lists $S^{\mathrm{NC}}$ and $S^{\mathrm{NN}}$ of partition statistics, in that order, and a list $\Sigma=\left(\sigma_{i}\right)$ of bijections establish a (typepreserving) bijection for a classical reflection group $W$ if, given either a classical noncrossing partition $\pi^{\mathrm{NC}}$ or a classical nonnesting partition $\pi^{\mathrm{NN}}$ for $W$, the other one exists uniquely such that $\sigma_{i}\left(s_{i}^{\mathrm{NC}}\left(\pi^{\mathrm{NC}}\right)\right)=s_{i}^{\mathrm{NN}}\left(\pi^{\mathrm{NN}}\right)$ for all $i$ (and furthermore $\pi^{\mathrm{NC}}$ and $\pi^{\mathrm{NN}}$ have the same type).

Suppose we have a tuple $\nu=\left(\nu_{1}, \ldots, \nu_{k}\right)$ with $k$ odd. Define the reordering

$$
\sigma_{B}(\nu)=\left(\nu_{1}, \ldots, \nu_{(k-1) / 2}, \nu_{(k+3) / 2}, \ldots, \nu_{k}, \nu_{(k+1) / 2}\right) .
$$

If $k$ is not odd then let $\sigma_{B}(\nu)=\nu$. Clearly $\sigma_{B}$ is bijective. For explicitness, the inverse for $k$ odd is given by

$$
\sigma_{B}^{-1}(\nu)=\left(\nu_{1}, \ldots, \nu_{(k-1) / 2}, \nu_{k}, \nu_{(k+1) / 2}, \ldots, \nu_{k-1}\right)
$$

and for $k$ even $\sigma_{B}^{-1}(\nu)=\nu$.

Theorem 2.9. The lists of statistics $(a, \mu, \nu)$ and $(a, \mu, \nu)$ establish a type-preserving bijection for $B_{n}$ via the bijections (id, id, $\sigma_{B}$ ).

Proof. We use the same procedures as in type $C$ to convert back and forth between classical nonnesting and noncrossing partitions, except that we must rearrange $\nu$ and handle the zero block appropriately, if it is present. When constructing a nonnesting partition we connect the incomplete switching blocks differently: in the notation of the dot-by-dot description, $P_{k}^{*}$ must be connected to $-P_{k}^{*}$ and the dot 0 , so that we connect $P_{i}^{*}$ to $-P_{k-i}^{*}$ for $1 \leq i<k$. The conditions of Corollary 2.8 , which as we noted above characterise type $B$ classical noncrossing and nonnesting partitions, don't depend on the order of $\nu$. So if tuples $a, \mu$, and $\nu$ satisfy them then so do $a, \mu$ and $\sigma_{B}(\nu)$ (or $\sigma_{B}^{-1}(\nu)$ ). Thus our statistics establish a bijection between $N C^{\mathrm{cl}}\left(B_{n}\right)$ and $N N^{\mathrm{cl}}\left(B_{n}\right)$.

Type is preserved, by the definition of $\sigma$ and the preceding discussion.

Figure 9 illustrates a pair of partitions related under the bijection. 


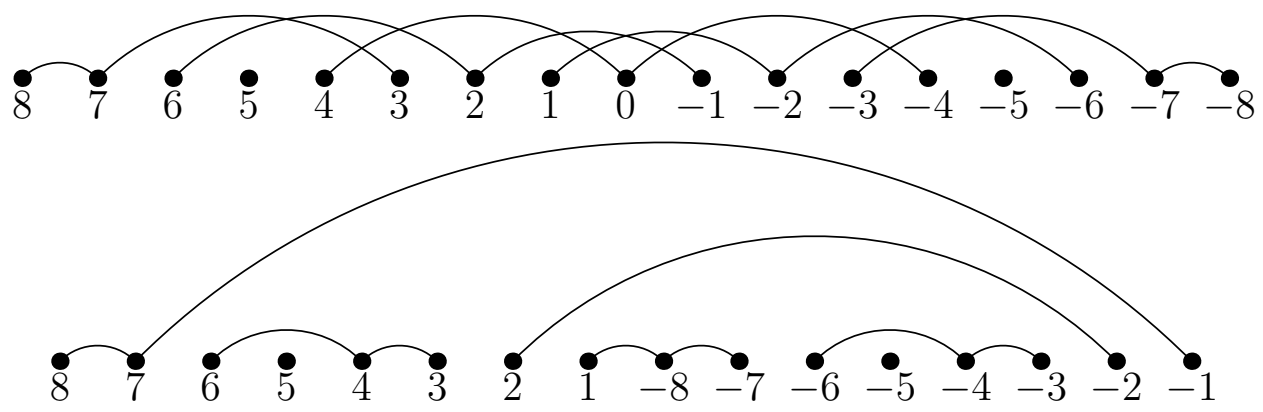

Figure 9 . The type $B$ nonnesting (top) and noncrossing (bottom) partitions corresponding to $a=(3,5), \mu=(3,1)$, and respectively $\nu=(1,2,1)$ and $\nu=(1,1,2)$. Note that $\sigma_{B}((1,1,2))=(1,2,1)$.

These correspond under the bijection of Theorem 2.9

2.5. Type $D$. The handling of type $D$ partitions is a further modification of our treatment of the foregoing types, especially type $B$.

In classical partitions for $D_{n}$, the elements \pm 1 will play much the same role as the element 0 of classical nonnesting partitions for $B_{n}$. So when applying the order $<_{\text {lp }}$ and the terminology of Definition 2.6 in type $D$ we will regard \pm 1 as being neither positive nor negative.

Given $\pi \in N N^{\mathrm{cl}}\left(D_{n}\right)$, define the statistics $a(\pi), \mu(\pi)$ and $\nu(\pi)$ as in type $B$. In this case we must have

$$
n-1=\sum_{i=1}^{m} \mu_{i}+\sum_{j=1}^{k} \nu_{j} .
$$

Let $R_{1}<_{\text {lp }} \cdots<_{\text {lp }} R_{l}$ be the blocks of $\pi$ which contain both a positive element and either 1 and -1 . It is clear that $l \leq 2$. Define the statistic $c(\pi)=\left(c_{1}, \ldots, c_{l}\right)$ by $c_{i}=R_{i} \cap\{1,-1\}$. To streamline the notation we'll usualy write $c_{i}$ as one of the symbols,,+- \pm . Observe that $\pi$ contains a zero block if and only if $c(\pi)=( \pm)$.

To get a handle on type $D$ classical noncrossing partitions, we will transform them into type $B$ ones. Let $N C_{\mathrm{r}}^{\mathrm{cl}}\left(B_{n-1}\right)$ be a relabelled set of classical noncrossing partitions for $B_{n-1}$, in which the parts $1, \ldots,(n-1)$ and $-1, \ldots,-n-1$ are changed respectively to $2, \ldots, n$ and $-2, \ldots,-n$. Define a map $C M: N C^{\mathrm{cl}}\left(D_{n}\right) \rightarrow$ $N C_{\mathrm{r}}^{\mathrm{cl}}\left(B_{n-1}\right)$, which we will call central merging, such that for $\pi \in N N^{\mathrm{cl}}\left(D_{n}\right)$, $C M(\pi)$ is the classical noncrossing $B_{n-1}$-partition obtained by first merging the blocks containing \pm 1 (which we've drawn at the center of the circular diagram) into a single part, and then discarding these elements \pm 1 . Define the statistics $a, \mu$ and $\nu$ for $\pi$ to be equal to those for $C M(\pi)$, where the entries of $a$ should acknowledge the relabelling and thus be chosen from $\{2, \ldots, n\}$.

These statistics do not uniquely characterise $\pi$, so we define additional statistics $c(\pi)$ and $\xi(\pi)$. The definition of $c(\pi)$ is analogous to the nonnesting case: let $R_{1}<_{\text {lp }} \cdots<_{\text {lp }} R_{l}$ be the blocks of $\pi$ which intersect $\{1,-1\}$, and define $c(\pi)=$ $\left(c_{1}, \ldots, c_{l}\right)$ where $c_{i}=R_{i} \cap\{1,-1\}$. Also define $\zeta(\pi)=\left(\zeta_{1}, \ldots, \zeta_{l}\right)$ where $\zeta_{l}=$ $\#\left(R_{l} \cap\{2, \ldots, n\}\right)$ is the number of positive parts of $R_{l}$.

Observe that $C M(\pi)$ lacks a zero block if and only if $c(\pi)=()$, the case that 1 and -1 both belong to singleton blocks of $\pi$. In this case $C M(\pi)$ is just $\pi$ with 
the blocks $\{1\}$ and $\{-1\}$ removed, so that $\pi$ is uniquely recoverable given $C M(\pi)$. Otherwise, $C M(\pi)$ has a zero block. If $c(\pi)=( \pm)$ this zero block came from a zero block of $\pi$, and $\pi$ is restored by resupplying \pm 1 to this zero block. Otherwise two blocks of $\pi$ are merged in the zero block of $C M(\pi)$. Suppose the zero block of $C M(\pi)$ is $\left\{c_{1}, \ldots, c_{j},-c_{1}, \ldots,-c_{j}\right\}$, with $0<c_{1}<\cdots<c_{j}$, so that $j=\sum_{i=1}^{l} \zeta_{l}$. By the noncrossing and symmetry properties of $\pi$, one of the blocks of $\pi$ which was merged into this block has the form $\left\{-c_{i+1}, \ldots,-c_{j}, c_{1}, \ldots, c_{i}, s\right\}$ where $1 \leq i \leq j$ and $s \in\{1,-1\}$. Then, by definition, $c(\pi)=(s,-s)$ and $\xi(\pi)=(i, j-i)$, except that if $j-i=0$ the latter component of each of these must be dropped. In this case the merged blocks of $\pi$ can be reconstructed since $c$ and $\xi$ specify $s$ and $i$.

Let a tagged noncrossing partition for $B_{n-1}$ be an element $\pi \in N C_{\mathrm{r}}^{\mathrm{cl}}\left(B_{n-1}\right)$ together with tuples $c(\pi)$ of nonempty subsets of $\{1,-1\}$ and $\xi(\pi)$ of positive integers such that:

(1) the entries of $c(\pi)$ are pairwise disjoint;

(2) $c(\pi)$ and $\xi(\pi)$ have equal length;

(3) the sum of all entries of $\xi(\pi)$ is the number of positive elements in the zero block of $\pi$.

Lemma 2.10. Central merging gives a bijection between classical noncrossing partitions for $D_{n}$ and tagged noncrossing partitions for $B_{n-1}$.

Proof. The foregoing discussion establishes that $C M$ is bijective. In view of this we need only check that the noncrossing property is preserved when moving between $\pi$ and $C M(\pi)$. In terms of bump diagrams, if $C M(\pi)$ is noncrossing $\pi$ is easily seen to be. For the converse, suppose $C M(\pi)$ has a crossing. This must be between the zero block $O$ and some other block $B$ of $C M(\pi)$, so that it is possible to choose $i, j \in B$ and $k \in O$ such that the segments $(i, j)$ and $(k,-k)$ within the bump diagram of $C M(\pi)$ cross. But these segments also cross in the bump diagram for $\pi$ and are contained within different blocks.

We show next that partitions are uniquely determined by the data we have associated with them.

Lemma 2.11. A classical nonnesting partition $\pi$ for $D_{n}$ is uniquely determined by the values of $a(\pi), \mu(\pi), \nu(\pi)$, and $c(\pi)$.

Proof. We reduce to the analogous facts for classical nonnesting partitions of types $B$ and $C$. There are slight variations in the behaviour depending on $c(\pi)$, so we break the argument into cases.

If $c(\pi)=()$, then dropping the elements \pm 1 from $\pi$ and relabelling $2, \ldots, n$, $-2, \ldots,-n$ to $1, \ldots, n-1,-1, \ldots,-(n-1)$ yields a nonnesting partition $\pi^{\prime}$ for $C_{n-1}$, and this is uniquely characterised by $a\left(\pi^{\prime}\right), \mu\left(\pi^{\prime}\right)$, and $\nu\left(\pi^{\prime}\right)$, which only differ from the statistics of $\pi$ by the relabelling in $a$.

If $c(\pi)=( \pm)$, then merging the elements \pm 1 into a single element 0 and relabelling $2, \ldots, n,-2, \ldots,-n$ to $1, \ldots, n-1,-1, \ldots,-(n-1)$ yields a nonnesting $B_{n-1}$-partition, and this is again uniquely characterised by $a\left(\pi^{\prime}\right), \mu\left(\pi^{\prime}\right)$, and $\nu\left(\pi^{\prime}\right)$, which only differ from the statistics of $\pi$ by the relabelling in $a$.

The cases $c(\pi)=(-)$ and $c(\pi)=(+,-)$ are carried under the exchange of +1 and -1 respectively to $c(\pi)=(+)$ and $c(\pi)=(-,+)$, so it suffices to handle only the latter two. 
We claim that, in these latter two cases, $\pi$ is itself a classical nonnesting partition for $C_{n}$. We will write $\pi^{\prime}$ for $\pi$ when we mean to conceive of it as an element $N N^{\mathrm{cl}}\left(C_{n}\right)$; in particular $\pi$ and $\pi^{\prime}$ will have different statistics. Since the ground set order for $N N^{\mathrm{cl}}\left(C_{n}\right)$ is a refinement of the order for $N N^{\mathrm{cl}}\left(D_{n}\right)$ in which only the formerly incomparable elements 1 and -1 in $\pi$ have become comparable in $\pi^{\prime}$, $\pi^{\prime}$ will be in $N N^{\text {cl }}\left(C_{n}\right)$ so long as no nestings involving edges of $G(\pi)$ terminating at 1 and -1 are introduced. By symmetry, if there is such a nesting, there will be one involving the edges $(i, 1)$ and $(j,-1)$ of $G\left(\pi^{\prime}\right)$ for some $i, j>1$. But the fact that $c(\pi)$ ends with + implies either $i>j$ or the edge $(j,-1)$ does not exist, so there is no nesting of this form.

When we readmit 1 and -1 as positive and negative elements, respectively, every nonswitching block of $\pi$ remains nonswitching in $\pi^{\prime}$, and every switching block of $\pi$ remains switching unless its only nonpositive element was 1 ; in this latter case -1 is likewise the only nonnegative element of its block, which happens iff $c(\pi)=(+)$.

Let $a(\pi)=\left(a_{1}, \ldots, a_{m}\right), \mu(\pi)=\left(\mu_{1}, \ldots, \mu_{m}\right), \nu(\pi)=\left(\nu_{1}, \ldots, \nu_{m}\right)$. In the case $c(\pi)=(-,+)$, we have

$$
\begin{aligned}
& a\left(\pi^{\prime}\right)=a(\pi) \\
& \mu\left(\pi^{\prime}\right)=\mu(\pi) \\
& \nu\left(\pi^{\prime}\right)=\left(\nu_{k}+1, \nu_{1}, \ldots, \nu_{k-1}\right) .
\end{aligned}
$$

That is, the block containing 1 is the greatest switching block of $\pi$ under $<_{\text {lp }}$ by assumption, but in $\pi^{\prime}$ where 1 is positive it becomes the first switching block. The other switching blocks are unchanged in number of positive elements and order, and nothing changes about the switching blocks. In case $c(\pi)=(+)$, the block containing 1 contains no other nonpositive element, so it becomes a nonswitching block, and in this case we get

$$
\begin{aligned}
& a\left(\pi^{\prime}\right)=\left(1, a_{1}, \ldots, a_{m}\right) \\
& \mu\left(\pi^{\prime}\right)=\left(\nu_{k}+1, \mu_{1}, \ldots, \mu_{m}\right) \\
& \nu\left(\pi^{\prime}\right)=\left(\nu_{1}, \ldots, \nu_{k-1}\right) .
\end{aligned}
$$

In either case $\pi^{\prime}$ is a classical nonnesting partition for $C_{n}$, and as such is determined by its statistics, but the translations (2.1) and (2.2) are injective so that $\pi$ is determined by its statistics as well.

Note that, when $c(\pi)$ is $(+)$ or $(-,+), \pi^{\prime}$ is an arbitrary noncrossing partition for $C_{n}$ subject to the condition that 1 is not the only positive element of its block. The cases $(+)$ and $(-,+)$ can be distinguished by whether $a\left(\pi^{\prime}\right)$ starts with 1 . Note also that the blocks of $\pi$ which contain one of the parts \pm 1 are exactly those described by the last $l$ components of $\nu(\pi)$, where $l$ is the length of $c(\pi)$.

All that remains to obtain a bijection is to describe the modifications to $\nu$ that are needed for correct handling of the zero block and its components (rather as in type $B)$. For a classical nonnesting partition $\pi$ for $D_{n}$, find the tuples $a(\pi), \mu(\pi)$, $\nu(\pi)=\left(\nu_{1}, \ldots, \nu_{k}\right)$, and $c(\pi)$. Let $\xi(\pi)$ be the tuple of the last $l$ entries of $\nu(\pi)$, 
where $l$ is the length of $c(\pi)$. Define

$$
\begin{aligned}
& \left(\hat{\nu}(\pi), \xi_{\text {inv }}(\pi), c_{\text {inv }}(\pi)\right) \\
& \quad= \begin{cases}\left(\left(\nu_{1}, \ldots, \nu_{k / 2-1}, \nu_{k-1}+\nu_{k}, \nu_{k / 2} \ldots, \nu_{k-2}\right),\left(\xi_{2}, \xi_{1}\right),\left(c_{2}, c_{1}\right)\right) & \text { if } l=2 \\
\left(\left(\nu_{1}, \ldots, \nu_{(k-1) / 2}, \nu_{k}, \nu_{(k+1) / 2} \ldots, \nu_{k-1}\right), \xi(\pi), c(\pi)\right) & \text { if } l=1 \\
(\nu(\pi), \xi(\pi), c(\pi)) & \text { if } l=0\end{cases}
\end{aligned}
$$

Define a bijection $\sigma_{D}$ by $\sigma_{D}(\nu(\pi), c(\pi))=\left(\hat{\nu}(\pi), \xi_{\text {inv }}(\pi), c_{\text {inv }}(\pi)\right)$. This gives us all the data for a tagged noncrossing partition $C M\left(\pi^{\prime}\right)$ for $B_{n-1}$, which corresponds via central merging with a noncrossing partition $\pi^{\prime}$ for $D_{n}$. Going backwards, from a noncrossing partition $\pi^{\prime}$ we recover a nonnesting partition $\pi$ by applying central merging, finding the list of statistics $(a(\pi), \mu(\pi),(\nu(\pi), c(\pi)))$ via the equality

$$
\left.\left(\nu(\pi), c_{\pi}\right)=\sigma_{D}^{-1}\left(\nu\left(\pi^{\prime}\right), \xi\left(\pi^{\prime}\right), c_{(} \pi^{\prime}\right)\right)
$$

(the other statistics remain equal) and using these statistics to make a nonnesting partition as usual. Type preservation is implied within these modifications of the statistics. When a zero block exists, the number of positive parts it contains is preserved because of the equality $\xi\left(\pi^{\prime}\right)=\nu(\pi)_{k}$ which holds in that case. Our handling of $\nu$ leaves the components corresponding to switching blocks not containing 1 or -1 unchanged, so the number of positive parts in these blocks is also preserved. The number of positive parts in the blocks containing 1 or -1 is preserved because $\xi\left(\pi^{\prime}\right)$ corresponds to $c\left(\pi^{\prime}\right)$ as the $l$ last entries of $\nu(\pi)$ correspond to $c(\pi)$ in all cases. The size of each nonswitching block is preserved in the statistic $\mu$, as in previous cases.

All in all, we have just proved the following theorem.

Theorem 2.12. The lists of statistics $(a, \mu,(\nu, \xi, c))$ and $(a, \mu,(\nu, c))$ establish a type-preserving bijection for $D_{n}$ via the bijections (id, id, $\left.\left(\sigma_{D}\right)^{-1}\right)$.

Figures 10 and 11 illustrate this bijection.

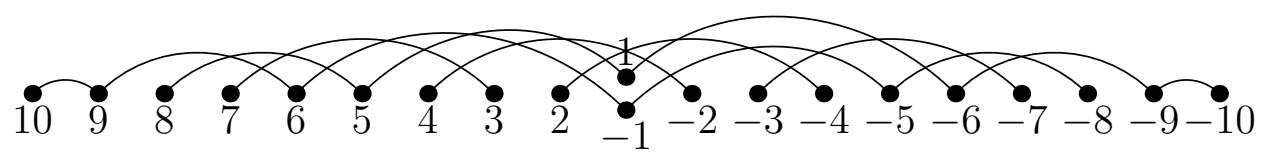

FIgURE 10. The $D_{10}$ nonnesting partition corresponding to $a=$ $(3), \mu=(2), \nu=(1,1,2,3), c=(+,-)($ so $\hat{\nu}=(1,5,1))$.

Finally we present a characterization of the values of $a, \mu, \nu$ and $c$ that describe type $D$ classical nonnesting partitions. As for noncrossing partitions, between our discussion of type $B$ and the definition of tagged partitions and Lemma 2.10, we have already presented all parts of the analogous result.

Corollary 2.13. Suppose we are given the tuples of positive integers $a=\left(a_{1}, \ldots, a_{m_{1}}\right)$, $\mu=\left(\mu_{1}, \ldots, \mu_{m_{2}}\right)$ and $\nu=\left(\nu_{1}, \ldots, \nu_{k}\right)$, and a tuple $c=\left(c_{1}, \ldots, c_{l}\right)$ of nonempty subsets of $\{1,-1\}$. Let $n>0$. Define $a_{0}=1$ and $\mu_{0}=2$. Then, $a, \mu, \nu$ and $c$ represent a classical nonnesting partition for $D_{n}$ if and only if

(1) $m_{1}=m_{2}=m$;

(2) $n-1=\sum_{i=1}^{m} \mu_{i}+\sum_{j=1}^{k} \nu_{j}$;

(3) $a_{i-1}<a_{i} \leq \sum_{k=0}^{i-1} \mu_{k}+\sum_{j=1}^{k} \nu_{j}$ for $i=1,2, \ldots, m$; 

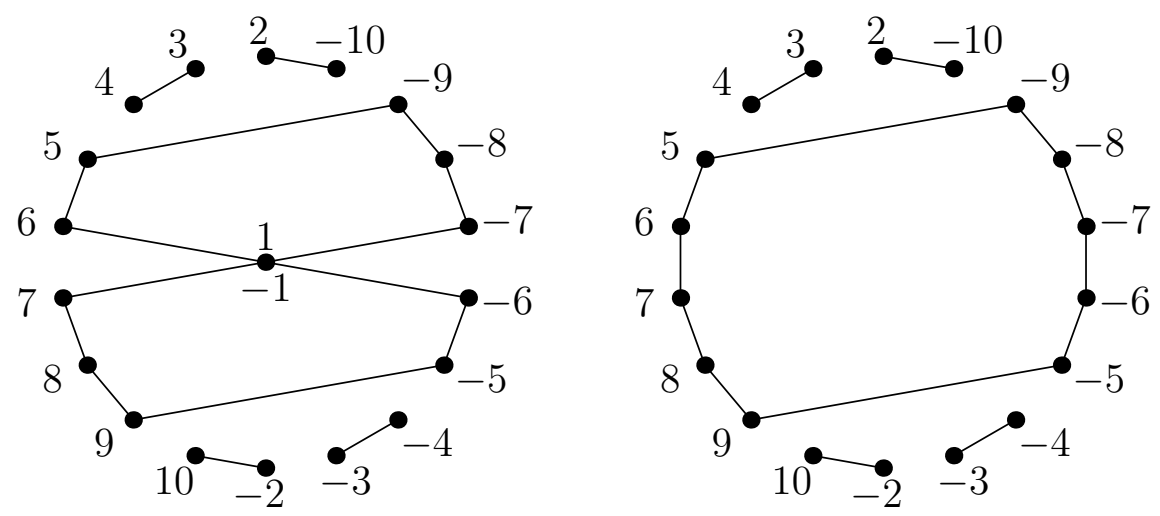

Figure 11. (left) The $D_{10}$ noncrossing partition corresponding to $a=(3), \mu=(2), \nu=(1,5,1), \xi=(3,2), c=(-,+)$. (right) The relabelled type $B$ noncrossing partition obtained via central merging.

(4) the entries of $c(\pi)$ are pairwise disjoint, so in particular $l \leq 2$;

(5) $k-l$ is even.

2.6. Proof of the central theorem. Using the preceding bijections we are now ready to establish our central result.

Proof of Theorem 2.2. When defining statistics and using the terminology of Definition 2.6 we consider positive integers as positive elements of blocks and negative integers as negative ones, without exception. Tag these new statistics with ${ }^{*}$ to distinguish them from the old statistics defined in Sections 2.2 through 2.5. Let $x^{\mathrm{cl}}$ be the classical partition representing $x$. Let $\eta^{*}\left(x^{\mathrm{cl}}\right)$ be the number of positive elements in the zero block of $x^{\mathrm{cl}}$. For any nonzero switching block $P$ of $x^{\mathrm{cl}}$, define the joint block

$$
S=\min _{<l_{\mathrm{p}}}(P,-P)
$$

and let $S_{1}<_{\mathrm{lp}} \cdots<_{\mathrm{lp}} S_{k^{\prime}}$ be the joint blocks of $x^{\mathrm{cl}}$. The number of joint blocks $k^{\prime}$ is half the number of nonzero switching blocks. Let $\vartheta_{+i}^{*}$ be the number of positive elements in $S_{i}$ and let $\vartheta_{-i}^{*}$ be the number of negative elements in $S_{i}$ and define the statistic $\vartheta^{*}\left(x^{\mathrm{cl}}\right)=\left(\left(\vartheta_{+1}^{*}, \vartheta_{-1}^{*}\right), \ldots,\left(\vartheta_{+k^{\prime}}^{*}, \vartheta_{-k^{\prime}}^{*}\right)\right)$. Finally, define as usual the statistics $a^{*}\left(x^{\mathrm{cl}}\right)=\left(a_{1}^{*}, \ldots, a_{m^{\prime}}^{*}\right)$ and $\mu^{*}\left(x^{\mathrm{cl}}\right)=\left(\mu_{1}^{*}, \ldots, \mu_{m^{\prime}}^{*}\right)$.

Let $y^{\mathrm{cl}}$ be the image of $x^{\mathrm{cl}}$ under the bijections of Theorems 2.4 2.12. There is a simple way to find a basis for $\operatorname{Fix}(x)$. From $x^{\mathrm{cl}}$ define a function $f: x^{\mathrm{cl}} \rightarrow \Psi^{n}$ in the following way. For any block $B$ of $x^{\mathrm{cl}}$, let

$$
f(B)=\operatorname{sgn}(B) \sum_{b \in B} \frac{b}{|b|} e_{|b|}
$$

where $\operatorname{sgn}(B)$ is +1 or -1 so that $f(B) \geq_{\operatorname{lex}} 0$ when $B$ is nonswitching and $-f(B) \geq_{\text {lex }} 0$ when $B$ is switching. The set $\beta:=f\left(x^{\mathrm{cl}}\right) \backslash\{0\}$ is the basis we are looking for, which we call the canonical basis of Fix $(x)$. 
For a (positive) nonswitching block $C_{i}$ of $x^{\mathrm{cl}}$, we have

$$
\begin{aligned}
\left|\underline{f\left(C_{i}\right)} \cap E\right| & =n+1-a_{i}^{*} \\
\#\left(f\left(C_{i}\right), 1\right) & =\mu_{i}^{*} \\
\#\left(f\left(C_{i}\right),-1\right) & =0 \\
\left|\underline{f\left(C_{i}\right)} \cap \beta\right| & =\left(m^{\prime}+1-i\right)+\left(k^{\prime}\right)
\end{aligned}
$$

For a joint block $S_{j}$ of $x^{\mathrm{cl}}$, we have

$$
\begin{aligned}
\left|\underline{f\left(S_{j}\right)} \cap E\right| & =0 \\
\#\left(f\left(S_{j}\right), 1\right) & =\vartheta_{-j}^{*} \\
\#\left(f\left(S_{j}\right),-1\right) & =\vartheta_{+j}^{*} \\
\underline{\mid f\left(S_{j}\right)} \cap \beta \mid & =j
\end{aligned}
$$

In any case, we have the equality

$$
\Gamma_{x}=\eta^{*}\left(x^{\mathrm{cl}}\right)
$$

Note that

$$
f\left(S_{1}\right)<_{\text {lex }} \ldots<_{\text {lex }} f\left(S_{k^{\prime}}\right)<_{\text {lex }} 0<_{\text {lex }} f\left(C_{m^{\prime}}\right)<_{\text {lex }} \ldots<_{\text {lex }} f\left(C_{1}\right)
$$

and that $m^{\prime}+k^{\prime}$ is the number of vectors in the ordered basis $\beta$. In fact

$$
\beta=\left\{f\left(S_{1}\right), \ldots, f\left(S_{k^{\prime}}\right), f\left(C_{m^{\prime}}\right), \ldots, f\left(C_{1}\right)\right\}
$$

Suppose $z$ is nonnesting or noncrossing partition of $W$ and suppose $\left\{v_{1}, \ldots, v_{p}\right\}$ is the canonical basis of $\operatorname{Fix}(z)$, ordered so that $v_{1}<_{\operatorname{lex}} \cdots<_{\operatorname{lex}} v_{n}$, which we don't know. Let $z^{\text {cl }}$ be the classical partition of $z$. Then, knowing the statistics $\mathcal{S}^{*}:=$ $\left(a^{*}, \mu^{*}, \vartheta^{*}, \eta^{*}\right)$ associated to $z^{\mathrm{cl}}$ allows us to recover the data in 2.3) through 2.5 associated to each of the $v_{i}$, and vice versa. Thus, the first step to reach our goal would be to prove that the bijections in Theorems 2.4 through 2.12 actually preserve the statistics $\mathcal{S}^{*}$. Any of the old statistics for $y^{\mathrm{c}}$ that is not mentioned in the following lines is trivially recovered from $\mathcal{S}^{*}$.

Assume without loss of generality that $x$ is a nonnesting partition, the other direction being completely analogous.

We begin with the case where $x$ is a nonnesting partition of $A_{n-1}$. The bijection of Theorem 2.4 clearly preserves $\mathcal{S}^{*}$. We have $a\left(y^{\mathrm{cl}}\right)=a^{*}\left(x^{\mathrm{cl}}\right)$ and $\mu\left(y^{\mathrm{cl}}\right)=\mu^{*}\left(x^{\mathrm{cl}}\right)$ so the uniqueness of $y^{\mathrm{cl}}$ is established directly from the statistics $\mathcal{S}^{*}$.

Suppose $x$ is an antichain for $C_{n}$. The statistics $a^{*}, \mu^{*}$ and $\eta^{*}$ are clearly preserved in Theorem 2.7. Also $\vartheta_{+i}^{*}=\nu_{i}$ and $\vartheta_{-i}^{*}=\nu_{k+1-i}$ so $\vartheta^{*}$ is also preserved. When there is a zero block we have $\nu_{(k+1) / 2}=\eta^{*}$ and this happens if and only if $\eta^{*}>0$. Therefore $y^{\mathrm{cl}}$ is characterized by $\mathcal{S}^{*}$.

Consider the case when $x$ is an antichain for $B_{n}$. Again, the statistics $a^{*}, \mu^{*}$ and $\eta^{*}$ are clearly preserved in Theorem 2.9. If there is no zero block we have $\vartheta_{+i}^{*}=\nu_{i}$ and $\vartheta_{-i}^{*}=\nu_{k+1-i}$. When there is a zero block we have

$$
\begin{array}{ll}
\vartheta_{+i}^{*}\left(x^{\mathrm{cl}}\right)=\nu_{i}\left(x^{\mathrm{cl}}\right) & \vartheta_{+i}^{*}\left(y^{\mathrm{cl}}\right)=\nu_{i}\left(y^{\mathrm{cl}}\right) \\
\vartheta_{-i}^{*}\left(x^{\mathrm{cl}}\right)=\nu_{k-i}\left(x^{\mathrm{cl}}\right) & \vartheta_{-i}^{*}\left(y^{\mathrm{cl}}\right)=\nu_{k+1-i}\left(y^{\mathrm{cl}}\right)
\end{array}
$$

but we also know that

$$
\nu_{i}\left(x^{\mathrm{cl}}\right)=\nu_{i}\left(y^{\mathrm{cl}}\right) \quad \text { and } \quad \nu_{k-i}\left(x^{\mathrm{cl}}\right)=\nu_{k+1-i}\left(y^{\mathrm{cl}}\right)
$$


so $\vartheta^{*}$ is preserved. There is a zero block if and only if $\eta^{*}>0$ and here we know in addition that $\nu_{k}\left(x^{\mathrm{cl}}\right)=\eta^{*}$ and $\nu_{(k+1) / 2}\left(y^{\mathrm{cl}}\right)=\eta^{*}$. Therefore $y^{\mathrm{cl}}$ is again characterized by $\mathcal{S}^{*}$.

We now consider the case when $x$ is an antichain for $D_{n}$. This part is divided into several subcases. Consider first when $c\left(x^{\mathrm{cl}}\right)=()$. Here, $x^{\mathrm{cl}}$ is a classical nonnesting partition for $B_{n}$ and its image $y^{\mathrm{cl}}$ under Theorem 2.12 is the unique classical noncrossing partition from Theorem 2.9 so the previous type suffices. We know $c\left(x^{\mathrm{cl}}\right)=()$ holds exactly when $a_{1}^{*}=1, \mu_{1}^{*}=1$ and $\eta^{*}=0$.

Suppose we have $c\left(x^{\mathrm{cl}}\right)=(+)$. Here the element +1 belongs to a nonswitching block of size $>1$. In the bijection of Theorem 2.12 the statistics $\mathcal{S}^{*}$ are preserved and this case is characterized by $a_{1}^{*}=1, \mu_{1}^{*}>1$ and $\eta^{*}=0$. Furthermore, on the noncrossing side we have

$$
\begin{aligned}
& a\left(y^{\mathrm{cl}}\right)=\left(\widehat{a}_{1}^{*}, a_{2}^{*}, \ldots, a_{m^{\prime}}^{*}\right) \\
& \mu\left(y^{\mathrm{cl}}\right)=\left(\widehat{\mu}_{1}^{*}, \mu_{2}^{*}, \ldots, \mu_{m^{\prime}}^{*}\right) \\
& \nu\left(y^{\mathrm{cl}}\right)=\left(\vartheta_{+1}^{*}, \ldots, \vartheta_{+k^{\prime}}^{*}, \mu_{1}^{*}-1, \vartheta_{-k^{\prime}}^{*}, \ldots, \vartheta_{-1}^{*}\right) \\
& \xi\left(y^{\mathrm{cl}}\right)=\left(\mu_{1}^{*}-1\right) \\
& c\left(y^{\mathrm{cl}}\right)=(+)
\end{aligned}
$$

so the uniqueness of $y^{\mathrm{cl}}$ is established directly from $\mathcal{S}^{*}$.

Suppose $c\left(x^{\mathrm{cl}}\right)=(-)$. The statistics $a^{*}, \mu^{*}$ and $\eta^{*}$ are preserved. To check that $\vartheta^{*}$ is preserved we have

$$
\begin{array}{ll}
\vartheta_{+1}^{*}\left(x^{\mathrm{cl}}\right)=1 & \vartheta_{+1}^{*}\left(y^{\mathrm{cl}}\right)=1 \\
\vartheta_{-1}^{*}\left(x^{\mathrm{cl}}\right)=\nu_{k}\left(x^{\mathrm{cl}}\right) & \vartheta_{-1}^{*}\left(y^{\mathrm{cl}}\right)=\nu_{\frac{k+1}{2}}\left(y^{\mathrm{cl}}\right) \\
\vartheta_{+i}^{*}\left(x^{\mathrm{cl}}\right)=\nu_{i-1}\left(x^{\mathrm{cl}}\right) & \vartheta_{+i}^{*}\left(y^{\mathrm{cl}}\right)=\nu_{i-1}\left(y^{\mathrm{cl}}\right) \quad \text { for } i>1 \\
\vartheta_{-i}^{*}\left(x^{\mathrm{cl}}\right)=\nu_{k-i}\left(x^{\mathrm{cl}}\right) & \vartheta_{-i}^{*}\left(y^{\mathrm{cl}}\right)=\nu_{k+1-i}\left(y^{\mathrm{cl}}\right) \quad \text { for } i>1
\end{array}
$$

However, we know the following equalities hold.

$$
\begin{gathered}
\nu_{k}\left(x^{\mathrm{cl}}\right)=\nu_{\frac{k+1}{2}}\left(y^{\mathrm{cl}}\right) \\
\nu_{i-1}\left(x^{\mathrm{cl}}\right)=\nu_{i-1}\left(y^{\mathrm{cl}}\right) \quad \text { and } \quad \nu_{k-i}\left(x^{\mathrm{cl}}\right)=\nu_{k+1-i}\left(y^{\mathrm{cl}}\right) \quad \text { for } i>1
\end{gathered}
$$

Hence, $\vartheta^{*}$ is indeed preserved. We also know that $c\left(x^{\mathrm{cl}}\right)=(-)$ if and only if $a_{1}^{*}>1, \vartheta_{+1}^{*}=1$ and $\eta^{*}=0$. Using the previous equations we may see that $\nu\left(y^{\mathrm{cl}}\right)$ is obtained uniquely from $\vartheta^{*}$, therefore $y^{\mathrm{cl}}$ is characterized by $\mathcal{S}^{*}$.

Suppose $c\left(x^{\mathrm{cl}}\right)=( \pm)$. Here it is easily seen that $\mathcal{S}^{*}$ are preserved. The characterization for the case is $\eta^{*}>0$ and the uniqueness of $y^{\mathrm{cl}}$ is also easily established.

Finally, consider the case when $l=2$ so either $c\left(x^{\mathrm{cl}}\right)=(+,-)$ or $c\left(x^{\mathrm{cl}}\right)=(-,+)$ holds. To start, suppose that $c\left(x^{\mathrm{cl}}\right)=(+,-)$. The bijection of Theorem 2.12 preserves $a^{*}, \mu^{*}$ and $\nu^{*}$ clearly. To see that $\vartheta^{*}$ is also preserved we need the more intricate equalities

$$
\begin{array}{llrl}
\vartheta_{+1}^{*}\left(x^{\mathrm{cl}}\right)=\nu_{k-1}\left(x^{\mathrm{cl}}\right)+1 & \vartheta_{+1}^{*}\left(y^{\mathrm{cl}}\right)=\xi_{2}\left(y^{\mathrm{cl}}\right)+1 \\
\vartheta_{-1}^{*}\left(x^{\mathrm{cl}}\right)=\nu_{k}\left(x^{\mathrm{cl}}\right) & \vartheta_{-1}^{*}\left(y^{\mathrm{cl}}\right)=\xi_{1}\left(y^{\mathrm{cl}}\right) \\
\vartheta_{+i}^{*}\left(x^{\mathrm{cl}}\right)=\nu_{i}\left(x^{\mathrm{cl}}\right) & \vartheta_{+i}^{*}\left(y^{\mathrm{cl}}\right)=\nu_{i}\left(y^{\mathrm{cl}}\right) \quad \text { for } i>1 \\
\vartheta_{-1}^{*}\left(x^{\mathrm{cl}}\right)=\nu_{k-1-i}\left(x^{\mathrm{cl}}\right) & \vartheta_{-1}^{*}\left(y^{\mathrm{cl}}\right)=\nu_{k+1-i}\left(y^{\mathrm{cl}}\right) \quad \text { for } i>1
\end{array}
$$


But we know from the handling of the statistics for type $D$ that

$$
\nu_{k-1}\left(x^{\mathrm{cl}}\right)=\xi_{2}\left(y^{\mathrm{cl}}\right),
$$

because of the function $\sigma_{D}$;

$$
\nu_{k}\left(x^{\mathrm{cl}}\right)=\xi_{1}\left(y^{\mathrm{cl}}\right),
$$

also because of the function $\sigma_{D}$; and

$$
\nu_{i}\left(x^{\mathrm{cl}}\right)=\nu_{i}\left(y^{\mathrm{cl}}\right) \quad \text { and } \quad \nu_{k-1-i}\left(x^{\mathrm{cl}}\right)=\nu_{k+1-i}\left(y^{\mathrm{cl}}\right) \quad \text { for } i>1 .
$$

This implies that $\vartheta^{*}$ is preserved in Theorem 2.12. Note that $c\left(x^{\mathrm{cl}}\right)=(+,-)$ or $c\left(x^{\mathrm{cl}}\right)=(-,+)$ occurs whenever none of the previous cases holds or whenever $a_{1}^{*}>1, \vartheta_{1}^{*}>1$ and $\eta^{*}=0$. Note also that we can obtain $a\left(y^{\mathrm{cl}}\right), \mu\left(y^{\mathrm{cl}}\right), \nu\left(y^{\mathrm{cl}}\right)$ and the number of positive and negative elements in the block containing +1 directly from $\mathcal{S}^{*}$, but we cannot characterize $y^{\text {cl }}$. This is because the information in $\mathcal{S}^{*}$ does not tell apart two noncrossing partitions $y_{1}^{\mathrm{cl}}$ and $y_{2}^{\mathrm{cl}}$ with identical statistics $a, \mu$ and $\nu$ but such that $\xi\left(y_{1}^{\mathrm{cl}}\right)=\xi_{\text {inv }}\left(y_{2}^{\mathrm{cl}}\right)$ and $c\left(y_{1}^{\mathrm{cl}}\right)=c_{\text {inv }}\left(y_{2}^{\mathrm{cl}}\right), y_{1}^{\mathrm{cl}}$ and $y_{2}^{\mathrm{cl}}$ have the same statistics $\mathcal{S}^{*}$. In particular $\vartheta_{+1}^{*}\left(y_{1}^{\mathrm{cl}}\right)=\vartheta_{+1}^{*}\left(y_{2}^{\mathrm{cl}}\right)$ and $\vartheta_{-1}^{*}\left(y_{1}^{\mathrm{cl}}\right)=\vartheta_{-1}^{*}\left(y_{2}^{\mathrm{cl}}\right)$. If additionally we require that the element with smallest absolute value $>1$ in the block containing +1 changes sign from $x^{\mathrm{cl}}$ to $y^{\mathrm{cl}}$, then this would be Theorem 2.12 This extends to all cases and types in the following way. For a joint block $S_{i}$ of $x^{\mathrm{cl}}$ with more than one positive element, we require that the element with smallest nonminimal absolute value in $S_{i}$ and the equivalent element in its image block $S_{i}^{\prime}$ of $y^{\text {cl }}$ have opposite signs. This new requirement is simply a necessary condition for noncrossing (or nonnesting) bump diagrams in all other cases and it was discussed in the proof of Theorem 2.7, so there is no loss or change in the previous analysis if we consider it as being part of the bijections. However this is tantamount to requiring that for any such block $S_{i}$, the product of the first two nonzero components in $f\left(S_{j}\right)$ and $f\left(S_{i}^{\prime}\right)$ is not equal. Clearly $S_{i}$ satisfies \# $\left(f\left(S_{j}\right),-1\right)>1$ and $\#\left(f\left(S_{j}\right), 1\right)>0$ and these inequalities are equivalent to the condition imposed on $S_{i}$.

Therefore, if we can prove that $\Omega_{x}=\beta$ in the general case where $x$ is a nonnesting or noncrossing partition of $W$, we will be done.

Suppose $x$ is a noncrossing or nonnesting partition and we are on step $i$ in the construction of $\Omega_{x}$. We have $e_{i}$ and we want to obtain $u_{i} \in \Psi^{n} \cap \operatorname{Fix}(x)$ with $\left\|u_{i}-e_{i}\right\|<_{\text {lex }}$-minimal.

Consider the case when $i$ belongs to a zero block of $x^{\mathrm{cl}}$. This means that $\pi^{i}(\operatorname{Fix}(x))=\{0\}$ where $\pi^{i}$ is the canonical projection on the $i$-th coordinate. If $v$ belongs to $\Psi^{n} \cap \operatorname{Fix}(x)$ then $\left\|v-e_{i}\right\| \geq_{\text {lex }} e_{i}$ because $\left\|v-e_{i}\right\|_{i}=1$. Hence $u_{i}=0$ and $u_{i}$ does not enter $\Omega_{x}$.

Now consider when $i$ does not belong to a zero block. We first prove the uniqueness of $u_{i}$. Suppose there exist two vectors $u_{i}$ and $u_{i}^{\prime}$ such that $\left\|u_{i}-e_{i}\right\|$ and $\left\|u_{i}^{\prime}-e_{i}\right\|$ are $<_{\text {lex }}$-minimal. This implies $\left\|u_{i}-e_{i}\right\|=\left\|u_{i}^{\prime}-e_{i}\right\|$ but then $\left(u_{i}\right)_{i}=\left(u_{i}^{\prime}\right)_{i}$ and $\left|\left(u_{i}\right)_{j}\right|=\left|\left(u_{i}^{\prime}\right)_{j}\right|$ for $j \neq i$. The $<_{\text {lex-minimality condition im- }}$ plies that $u_{i}=u_{i}^{\prime}=0$ or $\left(u_{i}\right)_{i}=\left(u_{i}^{\prime}\right)_{i}=1$. Suppose $\left(u_{i}\right)_{i}=\left(u_{i}^{\prime}\right)_{i}=1$ holds and suppose $\left(u_{i}\right)_{j}=-\left(u_{i}^{\prime}\right)_{j}$ for some $j \neq i$. Then $\left(u_{i}+u_{i}^{\prime}\right) / 2$ belongs to $\Psi^{n} \cap \operatorname{Fix}(x)$ and $\left\|\left(u_{i}+u_{i}^{\prime}\right) / 2-e_{i}\right\|<_{\operatorname{lex}}\left\|u_{i}-e_{i}\right\|=\left\|u_{i}^{\prime}-e_{i}\right\|$, a contradiction. Thus $u_{i}$ is unique. Again, the $<_{\text {lex }}$-minimality condition implies $u_{i}=0$ or $\left(u_{i}\right)_{i}=1$. If $u_{i}=0$ then it does not enter $\Omega_{x}$. Suppose there exists some $j<i$ such that $\left(u_{i}\right)_{j} \neq 0$. In this case $\left\|0-e_{i}\right\|<_{\text {lex }}\left\|u_{i}-e_{i}\right\|$ and we obtain a contradiction. Therefore, if $u_{i}$ enters $\Omega_{x}$ then $\left(u_{i}\right)_{j}=0$ for $j<i$ and $\left(u_{i}\right)_{i}=1$. The $<_{\operatorname{lex}}$-minimality condition shows that 
actually $u_{i}$ is the vector in $\Psi^{n} \cap \operatorname{Fix}(x)$ with the least number of nonzero components such that $\left(u_{i}\right)_{j}=0$ for $j<i$ and $\left(u_{i}\right)_{i}=1$. Now $u_{i}$ satisfies these conditions if and only if $i$ is the least nonzero component of $f(B)$ for some nonzero block $B$ of $x^{\mathrm{cl}}$ so that $u_{i}=f(B)$ or $u_{i}=-f(B)$ (according to whether $B$ is nonswitching or switching, respectively). But the sets

$$
S_{1}, \ldots, S_{k^{\prime}}, C_{1}, \ldots, C_{m^{\prime}}
$$

are pairwise disjoint and their minimal positive elements are all different, and $u_{i}$ (or $-u_{i}$ ) always enters $\Omega_{x}$, so we obtain a correspondence between the elements of $\Omega_{x}$ and $\beta$.

\section{REFERENCES}

[1] D. Armstrong, Generalized noncrossing partitions and combinatorics of Coxeter groups, preprint, arXiv:math.CO/0611106 v2.

[2] D. Armstrong, Braid groups, clusters, and free probability: an outline from the AIM workshop, January 2005.

[3] C. Athanasiadis, On noncrossing and nonnesting partitions for classical reflection groups, Electronic J. Combin. 5 (1998), R42.

[4] C. Athanasiadis and V. Reiner, Noncrossing partitions for the group $D_{n}$, SIAM J. Discrete Math. 18 (2004), 397-417.

[5] D. Bessis, The dual braid monoid, Ann. Sci. École Norm. Sup. (4), 36 (2003), 647-683.

[6] A. Björner and F. Brenti, Combinatorics of Coxeter groups, GTM 231, Springer, 2005.

[7] T. Brady and C. Watt, $K(\pi, 1)$ 's for Artin groups of finite type, in Proceedings of the conference on geometric and combinatorial group theory, Part I (Haifa 2000), Geom. Dedicata. 94 (2002), 225-250.

[8] R. W. Carter, Conjugacy classes in the Weyl group, Compositio Mathematica 25 no. 1 (1972), $1-59$.

[9] A. Conflitti, R. Mamede, On noncrossing and nonnesting partitions of type D, preprint, http://arxiv.org/abs/0905.4371

[10] J. Humphreys, Reflection groups and Coxeter groups, Cambridge University Press, Cambridge, 1990.

[11] C. Krattenthaler and T. W. Müller, Decomposition numbers for finite Coxeter groups and generalised non-crossing partitions, Trans. Amer. Math. Soc., to appear; preprint, arXiv:0704.0199 2 .

[12] G. Kreweras, Sur les partitions non croisées d'un cycle, Discrete Math. 1 (1972), 333-350.

[13] R. Mamede, A bijection between noncrossing and nonnesting partitions of types A and B, preprint, arXiv:0810.1422 1 .

[14] A. Postnikov and R. Stanley, Deformations of Coseter hyperplane arrangements, preprint, April 14, 1997.

[15] V. Reiner, Non-crossing partitions for classical reflection groups, Discrete Math. 177 (1997), 195-222.

[16] E. Sommers, B-stable ideals in the nilradical of a Borel subalgebra, Canad. Math. Bull. 48 (2005), 460-472.

[17] R. Stanley, Enumerative combinatorics, vol. 2, Cambridge University Press, Cambridge, 1997.

[18] Christian Stump, Non-crossing partitions, non-nesting partitions and Coxeter sortable elements in types A and B, preprint, arXiv:0808.2822 1 .

(finka@math.berkeley.edu) Alex Fink, Department of Mathematics, University of CalIFORNIA, BERKELEY CA, USA 94720

(b-iriart@uniandes.edu.co) Benjamin Iriarte Giraldo, Departamento de Matemáticas, Universidad de los Andes, Bogotá, Colombia 